\title{
Review
}

Aldino Viegas ${ }^{\mathrm{a}}$, Thibault Viennet ${ }^{\mathrm{a}}$ and Manuel Etzkorn*

\section{The power, pitfalls and potential of the nanodisc system for NMR-based studies}

DOI 10.1515/hsz-2016-0224

Received June 6, 2016; accepted July 19, 2016; previously published online July 23, 2016

\begin{abstract}
The choice of a suitable membrane mimicking environment is of fundamental importance for the characterization of structure and function of membrane proteins. In this respect, usage of the lipid bilayer nanodisc technology provides a unique potential for nuclear magnetic resonance (NMR)-based studies. This review summarizes the recent advances in this field, focusing on (i) the strengths of the system, (ii) the bottlenecks that may be faced, and (iii) promising capabilities that may be explored in future studies.
\end{abstract}

Keywords: membrane proteins; nanodiscs; NMR.

\section{Introduction}

The usage of the lipid bilayer nanodiscs (ND) system for nuclear magnetic resonance (NMR)-based studies has led to both enthusiasm and frustration in many labs in the recent years. In this review we will give an overview of the power as well as the pitfalls of this intriguing system and will discuss its potential for future applications.

In general, structural studies can be strongly dependent on the target protein's environment. As such, the

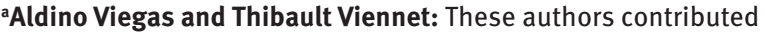
equally to this article.

*Corresponding author: Manuel Etzkorn, Institute of Physical Biology, Heinrich-Heine-University Düsseldorf, Universitätsstr. 1, D-40225 Düsseldorf, Germany; and Institute of Complex Systems, Forschungszentrum Jülich, Wilhelm-Johnen-Strasse, D-52425, Jülich, Germany, e-mail: manuel.etzkorn@hhu.de.

http://orcid.org/0000-0002-9796-3246

Aldino Viegas: Institute of Physical Biology, Heinrich-HeineUniversity Düsseldorf, Universitätsstr. 1, D-40225 Düsseldorf, Germany. http://orcid.org/0000-0003-1733-136X

Thibault Viennet: Institute of Physical Biology, Heinrich-HeineUniversity Düsseldorf, Universitätsstr. 1, D-40225 Düsseldorf, Germany; and Institute of Complex Systems, Forschungszentrum Jülich, Wilhelm-Johnen-Strasse, D-52425, Jülich, Germany.
}

influence of the environment on the protein structure as well as on the used experimental technique has to be considered. This is particularly true for the NMR-based investigation of membrane proteins, which require a suitable membrane mimicking environment to promote a protein conformation that is, on the one hand, representative for its native behavior and, on the other, compatible with the requirements of NMR spectroscopy. While a variety of different membrane mimetics for solution and/or solid-state NMR have been established (Popot, 2010; Raschle et al., 2010; Ding et al., 2013; Catoire et al., 2014; Liang and Tamm, 2016), the lipid bilayer nanodisc system distinctively combines various potentially advantageous properties including a native-like lipid surrounding, the absence of detergents, sample homogeneity, isolation of defined oligomeric states, and good accessibility of extra membranous domains. Noteworthy, these properties also make the ND system attractive as a tool for structural studies using non-NMR techniques [see e.g. Denisov and Sligar (2016) for a recent review and Gao et al. (2016) for a recent breakthrough in cryo EM studies of membrane proteins in NDs].

\section{History and background}

The nanodisc technology was first described by Sligar and co-workers (Bayburt et al., 1998, 2002) to support the study of the hepatic microsomal NADPH-cytochrome P450 reductase. The introduction of NDs as membrane mimetics provided a solution for studying membrane proteins in a native-like environment and some of the problems encountered in other mimetics. In particular, the absence of detergent molecules is one of the great advantages of this technology (Borch and Hamann, 2009; Bayburt and Sligar, 2010; Hiller and Wagner, 2012; Hagn et al., 2013).

Nanodiscs usually consist of an assembly of phospholipids held together by amphipathic apolipoproteins, normally called membrane scaffold proteins (MSPs), arranged in a discoidal bilayer (Figure 1) (Borch and Hamann, 2009). The MSPs are based on the sequence of the human serum apolipoprotein A-I (ApoA1), although other alternatives 

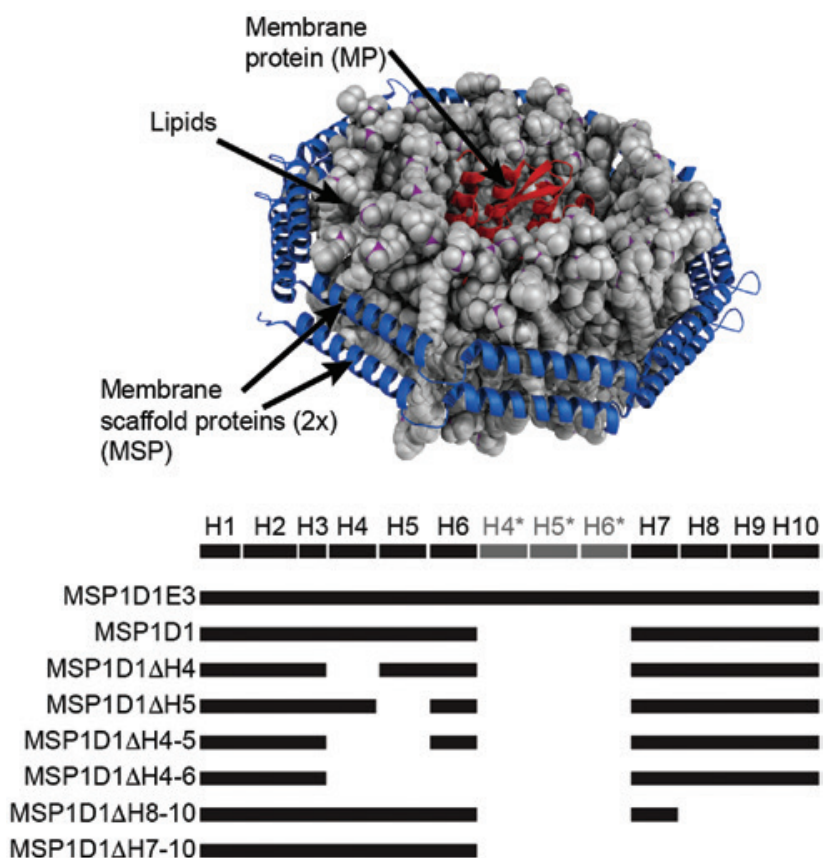

Figure 1: Schematic representation of the nanodisc system (top) and the different MSP constructs that have so far been used for NMR-based studies (bottom).

The gray $\mathrm{H}^{*}, \mathrm{H} 5^{*}$ and $\mathrm{H} 6^{*}$ helices are repeats in the MSP1D1E3 construct. The references for the different MSP construct can be found in Section, 'Choosing the MSP construct' (below).

exist (Chromy et al., 2007), and provide a hydrophobic surface facing the lipids, and a hydrophilic surface at the outside. This setup makes NDs highly soluble in aqueous solutions enabling the solubilization of embedded membrane proteins in the absence of detergents (Bayburt et al., 1998; Bayburt and Sligar, 2010). Depending on the used variant of MSP, the size of the nanodisc particles normally span between 6 and $17 \mathrm{~nm}$ in diameter (Grinkova et al., 2010; Wang et al., 2015). For NMR-related studies the most commonly used versions are the MSP1D1 and MSP1D1 $\Delta$ H5 (Hagn et al., 2013; Kucharska et al., 2015), which can result in soluble NDs with an overall particle mass below $200 \mathrm{kDa}$ that are in general applicable for modern NMR techniques (Shenkarev et al., 2010a; Klammt et al., 2012; Opella 2013).

\section{Structure of nanodiscs}

Nanodiscs are thought to closely resemble the natural early stage high density lipoproteins (HDL) involved in the reverse transport mechanism of cholesterol (Thomas et al., 2008; Shih et al., 2009). These discoidal HDLs have been shown to contain two apolipoproteins and roughly
160 phospholipids. However, no high-resolution structure of nanodiscs has been reported so far. Nevertheless, several models exist (Segrest 1977; Brouillette and Anantharamaiah, 1995; Brouillette et al., 2001; Phillips et al., 1997; Wu et al., 2007, 2009) with the most accepted one being the so-called 'double-belt' model (Segrest et al., 1999). This model is supported by the available crystal structures of lipid-free apolipoprotein A-I (Borhani et al., 1997; Ajees et al., 2006) as well as geometrical considerations (Segrest et al., 1999) and experimental data, such as cross-linking and mass-spectrometry (Bhat et al., 2007), small angle X-ray scattering (Skar-Gislinge et al., 2010), molecular dynamics (MD) simulations (Shih et al., 2005) and solid-state NMR data on the scaffold (Li et al., 2006) as well as on the lipid-protein correlations (Kijac et al., 2010) using PEG precipitated NDs. It seems plausible that the structure of the NDs is largely determined in the assembly process, which is driven mainly by hydrophobic interactions and protein-protein interactions, minimizing the hydrophobic exposure and maximizing the number of salt-bridges between the two MSPs (Shih et al., 2007a,b).

\section{Working with nanodiscs}

The usage of the nanodiscs system requires a number of steps that should be carefully considered to obtain optimal NMR insights into the desired target protein. While Figure 2 gives a schematic overview, selected steps will be discussed in more detail in the following.

\section{Choosing the MSP construct}

The geometry and size of NDs is defined by the scaffold protein MSP. Therefore, the choice of the MSP should be carefully and specifically evaluated for each application. Different MSP constructs have been engineered over the past 15 years, ranging from a length of 101 to 376 amino acid residues (Figure 1), and leading to diameters between 6 and $17 \mathrm{~nm}$. Originally two constructs were produced (Bayburt et al., 2002), MSP1 and MSP2, both forming discs with diameters around $9.5 \mathrm{~nm}$. The difference being that assembly requires either one MSP2 or two MSP1 for each nanodisc (MSP2 being a fusion of two MSP1 proteins, but leading to significantly lower yields). It is noteworthy that, albeit with lower yields and so far not explored properties for NMR structural studies, MSP2-based nanodisc carry an interesting potential due to their intrinsic architecture that features a closed circle of the scaffold 


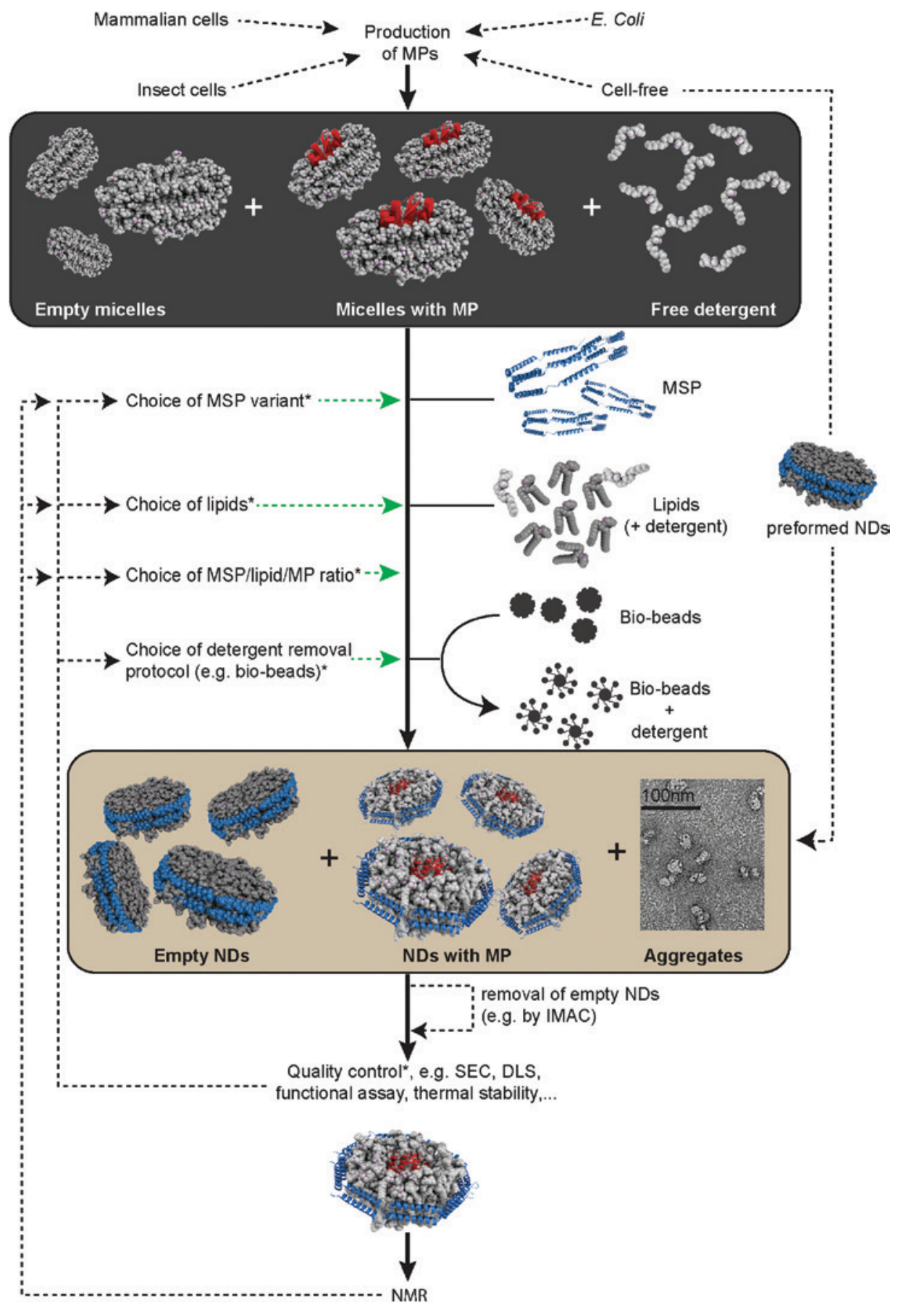

Figure 2: Schematic overview of the process to obtain NMR samples of ND-embedded membrane proteins. Black arrows indicate the normal process, dashed black arrows indicate optional/alternative options and green dashed arrows indicate optimization possibilities. * More details are given in the respective sections of the review.

protein potentially increasing stability and homogeneity. For MSP1 the first 12 amino acids were shown to not be involved in lipid binding and were thus removed, resulting in the so-called MSP1D1, which became the reference construct for production of NDs. Bigger constructs, called MSP1E1 to MSP1E3, were then introduced by extending the MSP1 by one to three helices, respectively (leading to diameters from 10.5 to $13 \mathrm{~nm}$ ) (Denisov et al., 2004). Even bigger NDs were designed using MSP2N2 and MSP2N3 with diameters around $17 \mathrm{~nm}$ (Grinkova et al., 2010). More recently, smaller NDs were developed with deletions of (parts) of the amphipathic helices in the center of MSP, e.g. MSP1D1 $\Delta \mathrm{H} 5$ and MSP1D1 $\Delta$ H4-6 (Hagn et al., 2013), or deletions of C-terminal helices (Puthenveetil and 
Vinogradova, 2013; Wang et al., 2015). Both were shown to form discs of 6-8 $\mathrm{nm}$ in diameter.

Most of the MSP constructs include an N-terminal polyhistidine tag for MSP purification and a TEV or Factor $\mathrm{X}$ cleavage site. Normally one would cleave the His-tag prior to assembly to allow separation of empty NDs from discs that have His-tagged membrane proteins embedded as well as to increase stability and NMR behavior (Kucharska et al., 2015). Nevertheless, in some cases it may be useful to not cleave the His-tag from the MSP, e.g. when purifying from native microsomal membrane (Civjan et al., 2003; Duan et al., 2004) or for immobilization of NDs for use of surface plasmon resonance (SPR) or other techniques using Nickel-NTA or anti-His antibody coupling (Shaw et al., 2007; Borch et al., 2008; Kawai et al., 2011). Other tags can be used as well for the same purpose, like C9 (Bocquet et al., 2015) or FLAG (Borch et al., 2008).

The size of the nanodiscs has to be chosen wisely according to the protein of interest. For instance, in the case of large integral membrane proteins, MSP1D1E3 is often preferred (Ritchie et al., 2009). Interestingly, varying the size of the NDs can also determine/influence the oligomeric state of the studied protein. For instance, it was shown that a minimal diameter of $12 \mathrm{~nm}$ was necessary for incorporating bacteriorhodopsin trimers, whereas smaller NDs could either result in heterogeneous or monomeric populations (Bayburt et al., 2006).

While most biochemical and biophysical techniques will not be influenced by the available range of ND sizes, solution NMR applications will strongly favor smaller NDs in order to reduce the rotational correlation time and thus increase spectral resolution and sensitivity. In this respect, the MSP1D1 $\triangle 5$ construct has been shown to be very effective (Hagn et al., 2013; Kucharska et al., 2015). On the contrary, for solid-state NMR applications, larger discs may be of interest (Kijac et al., 2007; Park et al., 2011; Ding et al., 2015a) making use of their magnetic alignment properties.

\section{Choosing the lipids}

The choice of lipids used to study MPs is of particular importance to obtain/maintain the target protein in its native conformation. In general, lipid composition of biological membranes is very diverse. It will not only change between different cell types or membrane types of one cell, but may also change over time, e.g. during the life cycle of the cell. Therefore, it is often difficult to define the exact native lipid composition for a given membrane protein. Nevertheless, the nanodisc system is compatible with a broad range of lipids and, theoretically, allows to precisely control lipid composition and generate large amounts of homogenous particles that are stable over time. In this respect it is a powerful tool to produce synthetic membranes tailored to adequately mimic a broad range of membrane properties including bilayer thickness, charge and fluidity. While standard phospholipids, including DMPC, DPPC or POPC are most commonly used for ND preparation, assembly has also been successful with phospholipid alkyl chains ranging from dilauroyl (DL, 12 carbons) to dielaidoyl (DE, 22 carbons), including unsaturated chains like dioleoyl (DO), and polar head groups varying from phosphocholine (PC, zwitterionic) to phospho-L-serine (PS, negatively charged) in different proportions up to 100\% negative charges (Shaw et al., 2007; Skar-Gislinge et al., 2010; Kawai et al., 2011; Roos et al., 2012; Shi et al., 2012; Bao et al., 2016; Rues et al., 2016). Moreover, usage of other types of lipids were reported including cardiolipin up to 10\% (Dalal et al., 2009; Roos et al., 2012; Hartley et al., 2013), ganglioside $\mathrm{G}_{\mathrm{M1}}$ up to $15 \%$ (Borch et al., 2008; Leney et al., 2014), sphingomyelin up to 30\% (Roy et al., 2015) or cholesterol up to 30\% (Bayburt et al., 1998; Proverbio et al., 2013). Although a broad range of lipids are in general compatible with the ND system, one should still keep in mind that when using lipid mixtures NDs are not necessarily formed with the statistical distribution of the lipids present during the assembly reaction. Instead, preferential interactions between lipids or between lipids and MSP might lead to a biased assembly. When using lipid mixtures, it is therefore advised to check the lipid content of the assembled ND. For example, this can be done by using radio-labeled lipids (Bayburt et al., 2002; Shaw et al., 2007), 'H NMR and HPLC-MS (Inagaki et al., 2012), analytical ultracentrifugation (Inagaki et al., 2013) or ion exchange chromatography (Lee et al., 2015).

Tuning the lipid content of nanodiscs was shown to improve MP activity (Boldog et al., 2007; Tsukamoto et al., 2010) as well as sample quality, stability and NMR behavior (Kucharska et al., 2015). This may be related to the role of unsaturation, cholesterol or head groups' negative charges that may help to stabilize the functional conformation of the membrane protein. For instance, NDs with a $20 / 80 \%$ PG/PC content provided largely increased NMR spectral quality as 100\% PC (Hagn et al., 2013; Ding et al., 2015b) (Figure 3D, E, unpublished data). While lipid screens should be used to optimize sample properties for NMR, they can, of course, also be used to study the effects of the surrounding lipids on the conformation, activity (Shaw et al., 2007; Inagaki et al., 2012) or affinity (Alami et al., 2007; Borch et al., 2008) of the inserted target protein. 
A
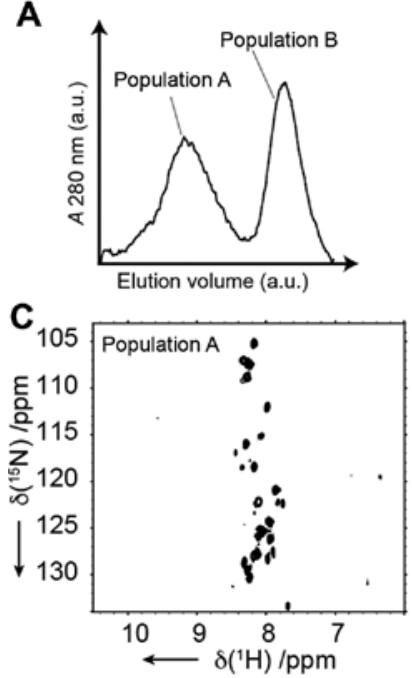

B
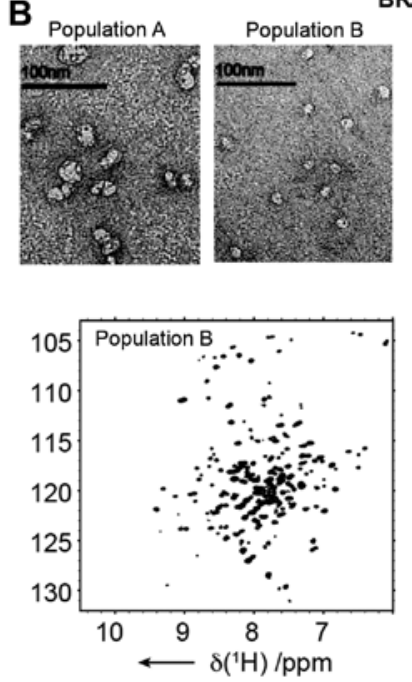

D

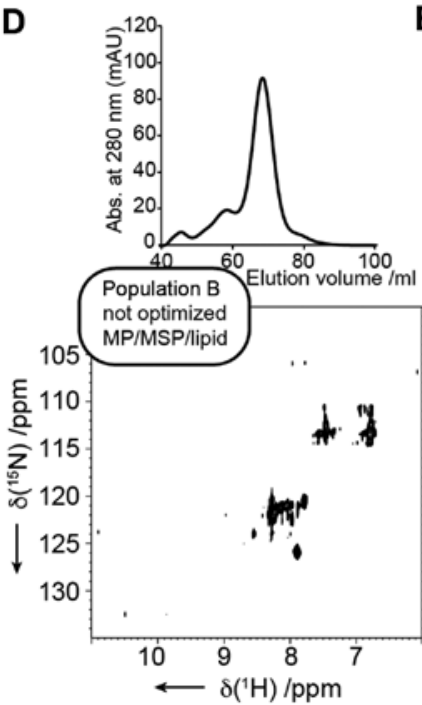

E

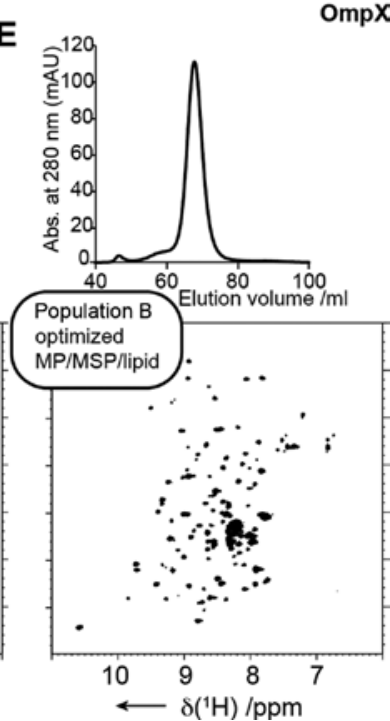

Figure 3: Role of the MSP/lipid/MP ratios and lipid composition. (A) SEC profile for a non-optimal MSP/lipid ratio showing the formation of two separate populations ( $A$ and $B$ ) of bR containing NDs. (B) Negative stained EM showing that population A consists of heterogeneously assembled ND (larger discs and clusters of discs) whereas population $B$ shows a very homogenous particle distribution. (C) Corresponding ${ }^{1} \mathrm{H}-{ }^{15} \mathrm{~N}$-TROSY-HSQC spectra showing that, while for population $B$ the signals are very well dispersed, for population $A$ only the peaks corresponding to the unstructured regions are visible. (Data in panels $\mathrm{A}-\mathrm{C}$ reprinted from Etzkorn et al., 2013, with permission from Elsevier.) (D and E) SEC profiles and corresponding ${ }^{1} \mathrm{H}-{ }^{15} \mathrm{~N}$-TROSY-HSQC spectra for the protein OmpX in NDs with different lipid composition and MSP:MP ratio, i.e. 100\% DMPC and 4:1 (D) and 80/20\% DMPC/DMPG and 6:1 (E).

As an alternative to synthetic lipid mixtures one can also directly use native membranes extracts. This can either be done by extracting lipids from the native cell membrane and using them as precursor for assembly with detergent purified MP (Alami et al., 2007; Boldog et al., 2007; Frauenfeld et al., 2011; Eggensperger et al., 2014), or by directly solubilizing protein-containing membrane fractions with detergent and replacing the detergent with suitable MSP. This approach has been successfully carried out for cytochrome $\mathrm{P} 450$ monooxygenase (Civjan et al., 2003) and the cytochrome $\mathrm{P} 450-\mathrm{P} 450$ reductase complex from insect cell culture (Duan et al., 2004). Note that native membrane extraction can also be carried out with amphipathic styrene-maleic acid (SMA) copolymer without the need for detergents (see Section 'Alternatives to MSP').

\section{Choosing the MSP-to-lipid and MSP-to-MP ratios}

The size, and thus the number of lipids a nanodisc contains, is largely determined by the chosen MSP (Denisov et al., 2004). Getting monodispersed NDs in high yield requires usage of a suitable ratio between the scaffold and the lipids during the assembly process. If too few lipids are present, smaller and poorly characterized particles can appear (Bayburt et al., 2006). On the contrary if too many lipids are present, bigger aggregated particles are formed (Bayburt et al., 2002; Shi et al., 2013). Additionally, the absolute concentrations of MSP and lipids during assembly are critical, and a minimum lipid concentration of $4 \mathrm{mM}$ is recommended (Bayburt et al., 2002; Leitz et al., 2006). The number of lipids per ND can often be quite accurately predicted from simple geometrical calculation since is it mostly determined by the total surface area and the area each lipid will occupy. Dividing the inner surface of the disc (calculated from the length of the used MSP and assuming a circular arrangement) by the surface of the chosen lipid is sufficient as a first step (Denisov et al., 2004; Ritchie et al., 2009); for instance, when forming empty NDs with MSP1D1, which has a single layer outer surface of $4400 \AA^{2}$, and DMPC, which has a head group surface of $57 \AA^{2}, 77$ lipids per single layer are needed (Bayburt and Sligar, 2010).

When incorporating membrane proteins in NDs, one has to compensate the bilayer area that will be occupied by the protein by reducing the respective number of lipids (Shaw et al., 2007; Ritchie et al., 2009). For example, one needs to remove around 37 DMPC molecules to compensate for bacteriorhodopsin insertion (Bayburt et al., 2006), or 50 POPC molecules for bovine rhodopsin (Bayburt et al., 2007). Exact lipid/MSP ratios may however also depend on other factors, such as the lipid binding behavior of the protein of interest and/or the phase of the resulting lipid 
bilayer. Thus, the best ratios for a specific MP-ND system should be empirically optimized around the expected values. For this, size exclusion chromatography (SEC) can be used as a readout to optimize towards homogenous particles of the expected size.

In addition to the MSP/lipid ratio, the MSP to target protein ratio (MSP/MP) can be critical to obtain nanodisc samples of good quality. The MSP/MP ratio can (partly) regulate the number of MPs per nanodisc. Increasing the MSP/MP ratio will normally increase the propensity to assemble NDs with no or only one inserted MP. Previous studies have used MSP/MP ratios ranging from 2:1 for the cytochrome $\mathrm{P} 450$ reductase to $150: 1$ for the $\beta 2$-adrenergic receptor (Bayburt and Sligar, 2003; Baas et al., 2004; Leitz et al., 2006; Bayburt et al., 2007; Boldog et al., 2007; Shaw et al., 2007; Whorton et al., 2007; Shi et al., 2012). For NMR structural studies a monomeric state of the target protein is often desired, which can be obtained with a high (>6-fold) excess of MSP over the target membrane protein and/or the choice of a MSP construct that prevents insertion of multiple proteins due to limited surface area. When lower MSP/MP ratios are used one should keep in mind that the assembly of native oligomers is not always straightforward because membrane orientation of the inserted proteins may differ and/or lateral diffusion could be restricted, thus limiting reorientation in small discs. Therefore, it may be beneficial to assemble native oligomers before insertion (e.g. by detergent extraction of native membrane fragments). Previous studies have, however, succeeded in the preparation of NDs containing defined multimeric states of membrane proteins. For instance, by varying the MSP/MP ratio, formation of dimers, trimers or even trimers of dimers has been observed (Bayburt et al., 2007; Boldog et al., 2007; Mi et al., 2008; Kawai et al., 2011). Insertion of bR trimers in a homogenous population of NDs was successfully obtained with a ratio of 2:3 (Bayburt et al., 2006; Tsukamoto et al., 2010; Kedrov et al., 2013; Shi et al., 2013). In addition to the study of oligomeric states of one membrane protein, the characterization of the interaction between two different MPs inside a single ND was also demonstrated with cytochrome P450 and its cytochrome $\mathrm{P} 450$ reductase starting from cell culture microsomes (Duan et al., 2004).

Noteworthy, instead of solely relying on the MSP/MP ratio to statistically drive the insertion of a desired multimeric state, which is often found to be randomly oriented in NDs (Banerjee et al., 2008), it has been shown that defined oligomeric states (dimers and trimers) in a defined orientation can be inserted into NDs by making use of DNA origami (Raschle et al., 2015) (see Section 'Perspectives/conclusion' for more details).
Figure 3 shows some of our experimental data in respect to the role of the $\mathrm{MSP} / \mathrm{lipid} / \mathrm{MP}$ ratio. Using a non-optimal MSP/lipid ratio led to the formation of two populations of bR containing NDs that can be visualized and separated by SEC (Figure 3A). Negative stained Electron microscopy (EM) (Figure 3B) shows that population A consisted of heterogeneously assembled ND (larger discs and clusters of discs) whereas population B shows a very homogenous particle distribution. Interestingly, as evident by the characteristic color of the samples, both populations contain correctly folded bR. Even though many biochemical and biophysical techniques will hence show a similar behavior of both populations, they have a dramatically different NMR behavior. While the smaller discs show a very well dispersed ${ }^{1} \mathrm{H}^{-15} \mathrm{~N}$-TROSY-HSQC spectrum that nearly resolves all residues in $\mathrm{bR}$, the larger discs (population A) only show few peaks at positions characteristic for unstructured proteins. In fact, for this population only the unstructured regions of bR (in particular the termini) are visible by conventional solution NMR spectroscopy (Etzkorn et al., 2013). Optimizing the $\mathrm{MSP} /$ lipid ratio resulted in generation of $>90 \%$ of population B (data not shown).

\section{Removing detergent}

Although often neglected, an appropriate detergent removal protocol plays a key role in the ND's auto-assembly reaction. As such, detergent removal does not only initiate the ND assembly process out of a mixed solution of proteins, lipids and detergents but can also determine the speed of the assembly reaction. Before nanodisc assembly not only the MP of interest but also the chosen lipids are solubilized with detergent (Figure 2). Sodium cholate has been shown to be an efficient detergent for phospholipid solubilization (Bayburt and Sligar, 2010) but sodium dodecylsulfate (SDS) or other detergents can be used as well (D’Antona et al., 2014). In some cases the same detergent can be used for resuspending the lipids and solubilizing the membrane proteins (Bayburt and Sligar, 2003; Reichart et al., 2016), e.g. when SDS-solubilized bacterioopsin is directly refolded into NDs (Etzkorn et al., 2013), but often milder detergents are used to directly transfer a folded protein conformation into the NDs. Mixed detergent approaches with lipids resuspended in cholate and protein solubilized in maltosides (Leitz et al., 2006; Kawai et al., 2011; Mitra et al., 2013), glycosides (Bayburt et al., 2007; Boldog et al., 2007; Shi et al., 2012), polyethylene glycol (Brij) (Mi et al., 2008), digitonin (Eggensperger et al., 2014), CHAPS (Bayburt and Sligar, 2010), Triton 
X-100 (Bayburt et al., 2006; Mi et al., 2008), Emulgen 913 (Baas et al., 2004), LDAO (Raschle et al., 2015) and FOS-12 micelles (Hagn et al., 2013; Bao et al., 2016) have been successful.

To prevent interference of residual detergent molecules and release the full potential of the ND system as a detergent free membrane mimetic, an extensive detergent removal should be carried out. The two most common ways of detergent removal are dialysis and adsorption by porous polystyrene beads, known as bio-beads. While the removal efficiency will be detergent dependent (in particular dependent on the critical micelle concentration), as a rule of thumb a dialysis against three times 1000 -fold volume of detergent free buffer or addition of $20-50 \%$ w/v beads are often used (Bayburt et al., 2002; Baas et al., 2004). Noteworthy, (particularly small) membrane proteins might themselves be adsorbed to the dialysis membrane or the bio-beads. In these cases, NDs can also be assembled by immobilizing the protein on agarose beads using, for instance, a His-tag (Katayama et al., 2010). Immobilization can also be used to wash the detergent out of the system, e.g. by using a conventional IMAC setting.

The rate of detergent removal determines the time scale of assembly and thus is of critical importance. While so far the exact mechanism is not fully understood, it may be pictured as a competition process between detergents and MSP as a stabilizing agent for the lipids. In this process, the absolute and relative concentrations of reactants, as well as the detergent removal rate, will be the factors that determine the overall yield and the sample homogeneity of the resulting NDs (Denisov et al., 2004). When MPs are to be inserted, the rate of detergent removal can also influence their oligomeric state. For instance, when monomers should be inserted into single NDs, the rate of detergent removal should be faster than the rate of oligomerization. In general the use of polystyrene beads with gentle agitation for a few hours produces well suitable detergent removal rates (Bayburt et al., 2006; Inagaki et al., 2012). However, also prolonged dialysis with two or three changes of buffer, or overnight use of adsorbent beads without agitation are common procedures. For some systems we have experienced that too fast detergent removal resulted in an increase of protein aggregates and we therefore commonly use a two steps protocol of $10-20 \% \mathrm{w} / \mathrm{v}$ bio-beads (first step overnight without shaking, second step 2-3 h with shaking after bio-beads exchange). In some cases also the use of a detergent-removing gel is preferred over dialysis which is a slower process (Banerjee et al., 2008; Nasvik Ojemyr et al., 2012).
Another important factor to consider is the temperature during ND assembly. The two main reasons for this are: (i) it will have an effect on the rate of detergent removal (see above) and (ii) it will determine the lipid bilayer phase. Usually it is recommended to carry out the reaction just below the phase transition temperature of the lipids which favors formation of the nanoscale bilayers. For example, temperatures of $38^{\circ} \mathrm{C}$ for DPPC $\left(T_{m}=41^{\circ} \mathrm{C}\right)$, room temperature for DMPC $\left(T_{m}=28^{\circ} \mathrm{C}\right)$ or ice water for POPC $\left(T_{m}=-2^{\circ} \mathrm{C}\right)$ are commonly chosen (Bayburt et al., 1998; Denisov et al., 2005; Leitz et al., 2006). When working with lipid mixtures in the ND assembly process, one should also consider that phase transition temperatures of the individual lipids may differ, which often results in a rather broad phase transition between the individual transition temperatures, indicative of lipidlipid interactions, in the case of mixed liposomes. In this respect the assembly temperature may be used to either promote (all lipids are in same phase) or impede (different phases for different lipids) homogenous mixing and insertion of different lipids in NDs. Note that heating (gelphase) assembled ND above the lipid phase transition can lead to reorganization of the lipids which may or may not be desired. Also note that it has been shown before that the phase transition properties of lipids are different in NDs as compared to liposomes (Shaw et al., 2004; Denisov et al., 2005).

\section{Quality control}

NMR spectral quality will depend on the homogeneity and structural integrity of the inserted membrane protein as well as the homogeneity of the assembled NDs. Therefore, it is of central importance to check basic biophysical properties after the assembly process. Standard procedures can include SDS-polyacrylamide gel electrophoresis (SDS-PAGE) to check the MSP/MP ratio (after removal of empty NDs), analytical SEC and/or DLS measurements to check particle sizes and distribution. Table 1 summarizes common pitfalls that may result in low quality data.

In any case, a preparative SEC step, to pool only the homogenous (small) NDs, is always recommended before NMR measurements. In general, a decent SEC profile is a necessary condition to obtain good NMR data and can therefore be used as an initial readout for an iterative optimization of the MSP/lipid/MP ratio or the detergent removal protocol (Figure 2). Although necessary, a good SEC profile may not be sufficient to obtain good NMR spectra (Figure 3D). In this respect it may be useful to 
Table 1: Common pitfalls that may result in low quality data.

\begin{tabular}{lll}
\hline Pitfalls & Effect/detection & Potential solution \\
\hline Not functional MP & - Functional assay & - Optimized refolding \\
& - Incorporation into ND (denatured & - Detergent and lipid screen \\
Wrong MP/lipid/ & MP normally do not insert into NDs) & - Co-translational ND incorporation in cell-free expression systems \\
MSP ratio & - Not homogenous SEC profile & - Geometrical consideration \\
& - SDS-PAGE band ratios MP/MSP & - Empirical optimization \\
Not intact lipids & - Bad HSQC spectra & \\
& - Bad reproducibility & - Pre-warming cold chloroform stocks before opening \\
& - Ice crystals in frozen chloroform & - Avoid contaminations with air, water, plastic tips, parafilm, etc. \\
Wrong MSP & & - Purchase smaller aliquots \\
construct & - Bad HSQC spectra & - Geometrical consideration \\
Wrong detergent & - Sample instability & - Empirical optimization \\
removal & - SEC profile & - Step-wise addition of smaller quantities of bio-beads \\
& - SDS-PAGE & - Usage of dilution/dialysis when (small) MPs are absorbed by bio-beads \\
& & - MP immobilization using e.g. Ni-NTA beads \\
\hline
\end{tabular}

optimize the lipid content directly using a NMR readout. Noteworthy, while it is possible to use a $1 \mathrm{D}^{1} \mathrm{H}$ spectrum as readout (e.g. a $1 \mathrm{D}$ version of a ${ }^{15} \mathrm{~N}$-filtered TROSY-HSQC) one should not optimize towards maximum signal intensity of the strongest peaks (since these result most likely from extra membranous residues like loops and termini). Instead one should optimize towards the appearance of (often considerably weaker) peaks on regions outside the random coil region (Figure 4). If peaks of unfolded, solvent-accessible protein regions are too intense and overshadow the membrane embedded protein regions, higher $\mathrm{pH}$ values and temperatures can be used to increase water exchange in the water accessible regions, effectively reducing the respective NMR signals.

In general, also optimization towards increased thermal stability (e.g. by thermal shift assays, differential scanning calorimetry, intrinsic tryptophan fluorescence, CD spectroscopy, etc.) can be useful, since spectral quality will also significantly increase with increased temperature during measurement (Figure 4). Note that the stability of empty NDs is in general very high, with denaturation temperatures well above $80^{\circ} \mathrm{C}$, so transitions detected below $70^{\circ} \mathrm{C}$ should normally report on the inserted MPs.

If nanodiscs with a defined lipid mixture need to be assembled, it may also be useful to check the actual composition of the assembled discs. In cases where different charges should be inserted this can be done using ion exchange chromatography (Lee et al., 2015). Of course NMR measurements can and should also be used to assess the actual lipid composition. For this, quantitative $1 \mathrm{D}$ and/or $2 \mathrm{D}{ }^{1} \mathrm{H}$ spectra can be recorded. Due to the specific relaxation properties of different lipid regions (e.g. solvent accessible head groups vs. membrane

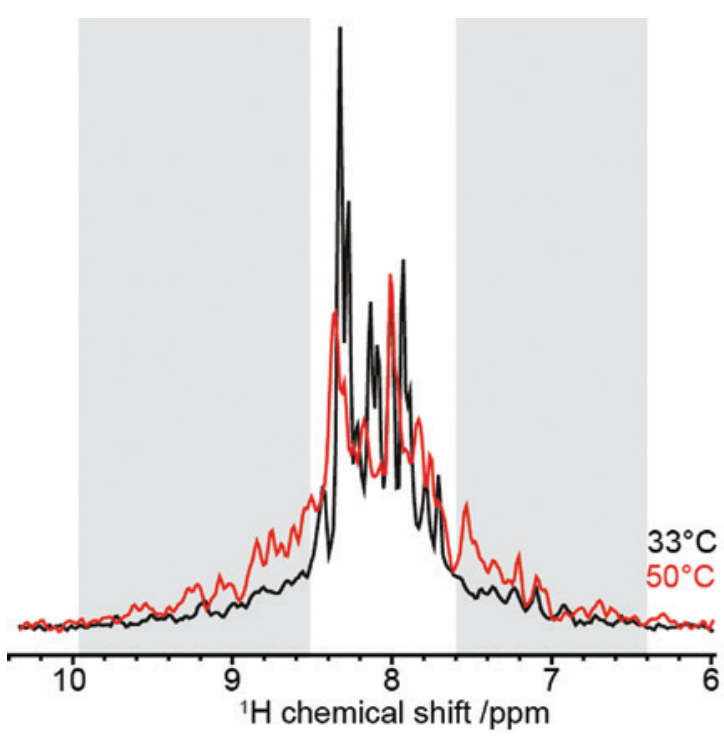

Figure 4: Optimization of ${ }^{1} \mathrm{H}-\mathrm{NMR}$ spectral properties. $1 \mathrm{D}$ versions of $\mathrm{a}^{15} \mathrm{~N}$-filtered TROSY-HSQC for bR in NDs formed with MSPD1 recorded at $33^{\circ} \mathrm{C}$ (black) and $50^{\circ} \mathrm{C}$ (red) are shown. When optimizing the conditions for NMR data acquisition, one should focus on the appearance of peaks on regions outside the random coil region (highlighted gray) instead of trying to maximize the intensity of the unfolded and solvent-accessible regions. Note that while the maximum signal intensity is higher for the $33^{\circ} \mathrm{C}$ spectrum the overall NMR quality is significantly better at $50^{\circ} \mathrm{C}$. The resulting $2 \mathrm{D}$ spectra can be found in the Supplementary Figure S3g,h of Etzkorn et al. (2013).

embedded hydrocarbon chains) the quantitative analysis of peak volumes however needs to be interpreted with care and may only be representative if peaks from different lipids with similar relaxation properties are compared.

It is noteworthy that when assembling NDs from SDS solubilized bacterioopsin and SDS-solubilized lipids, we 
observed that about 1-2 SDS molecules insert into the lipid bilayer (Etzkorn et al., 2013). While in this particular case no direct effect of SDS were visible, one should keep in mind that the assembled NDs system may also include small amounts of the used detergent in a membrane embedded (not in a soluble monomeric) form.

\section{Alternative expression systems and initial MP solubilization}

In general, when membrane proteins are recombinantly expressed in bacteria or in eukaryotic cell culture, solubilization and purification are regularly carried out using detergent micelles. The detergent step can however be detrimental to some MPs and can result in low yields of soluble, purified and functional MP.

As an alternative, cell-free expression (CFE) can be of great use for expression of various types of membrane proteins (Schwarz et al., 2007; Yang et al., 2011). CFE can be carried out with prokaryotic (E. coli) or eukaryotic (wheat germ, rabbit reticulocytes) extracts (Katzen et al., 2008) and can enable co-translational folding into membrane mimetic. Due to the open nature of the CFE systems, the addition of different membrane-mimicking systems (bicelles, liposomes, amphipols, etc.), including NDs is possible (Rajesh et al., 2011; Etzkorn et al., 2013; Proverbio et al., 2013; Roos et al., 2013). In addition to membrane mimetics, precursors and cofactors that can improve yield and folding as well as a wide range of isotopically labeled compounds can also be added to improve NMR insights (Lyukmanova et al., 2012; Etzkorn et al., 2013).

Co-translational incorporation of membrane proteins in NDs during CFE can be realized either by addition of preformed empty NDs to the reaction mixture (Katzen et al., 2008; Yang et al., 2011; Roos et al., 2012; Etzkorn et al., 2013; Rues et al., 2016), by addition of the individual components in a continuous exchange CFE chamber that can directly act as detergent removal dialysis chamber or by co-expression of the target protein and the MSP in a single reaction (Cappuccio et al., 2008; Gao et al., 2012). For both the addition of preformed empty NDs to the reaction mixture and the co-expression of the target protein and the MSP in a single reaction, the comparison between the fraction of solubilized membrane protein with the total membrane protein revealed rather high yields (between 50 and $90 \%$ ) of soluble and functional protein for several MPs (Cappuccio et al., 2008; Lyukmanova et al. 2012; Roos et al., 2012). However, for more challenging proteins, such as G protein-coupled receptors (GPCRs), co-translational incorporation of receptor into NDs resulted in lower yields (below 1\%) for not stabilized receptor and yields in the range of $0.8-30 \%$ of functional protein in NDs for thermostabilized receptors (Leitz et al., 2006; Rues et al., 2016). A broad range of ND types, with various sizes and lipid contents, have been reported in this context (Lyukmanova et al., 2012; Roos et al., 2012; Rues et al., 2016). The short time needed and the ease of adding different reactants to small scaled CFE make this approach suitable for the screening of various types of effects, including the study of membrane insertion itself (Roos et al., 2012).

However, despite the apparent benefits, the lack of a native membrane insertion and/or chaperon system that is either not inherent to the expression system (e.g. in the case of E.coli extract) or was separated from the (soluble) expression machinery (e.g. in the extract preparation) can still lead to inhomogeneous (Cappuccio et al., 2008) or inactive (Yang et al., 2011) samples. Nevertheless, tuning of the membrane mimetic properties (Roos et al., 2012) or addition of a translocon system, chaperones, signal sequences and cofactors could overcome this issue in some cases (Roos et al., 2013).

\section{Alternatives to MSP}

The nanodisc technology invented by Sligar and co-workers is based on the human ApoA1 protein and its ability to assemble into high density lipoprotein particles and the above discussion focused on MSP constructs that are derivatives of this protein. However, the use of ApoA1 from other species, like zebra fish (zap1), has also been reported (Banerjee et al., 2008). Additionally, alternatives to ApoA1 have also been used, including ApoE4 and the lipophorin ApoLpIII (Chromy et al., 2007). The truncated mutant ApoE422K provides good nanolipoprotein formation of around $15 \mathrm{~nm}$ in diameter and was used for incorporation of MPs during cell-free expression (Katzen et al., 2008) and solubilization of an active hyperthermophile hydrogenase (Baker et al., 2009). Moreover, formation of similar type of lipoproteoparticles (termed Salipro) has been reported using saposin A as a scaffold. This system was applied to the study of channels (T2), peptide transporters (PepT) and HIV spikes (gp) from viral-like particles (Frauenfeld et al., 2016).

The different apolipoproteins used as scaffold normally assemble into particles of 6-17 $\mathrm{nm}$ in diameter. However, for applications like self-alignment in the magnetic field (in order to extract residual dipolar couplings), larger discs also formed using a 14-residue class A 
amphipathic $\alpha$-helical peptide as scaffold and a diameter of roughly $30 \mathrm{~nm}$ (called macrodiscs), were used (Anantharamaiah et al., 1985; Park et al., 2011). It appears that usage of this scaffold peptide is possible for a broad range of particle sizes as it is not only defined by the length of the peptide but also by the scaffold to lipids ratio. Comparable effects were also seen using a similar 22-residue peptide (Zhang et al., 2016).

An additional alternative method has been developed, entitled lipodisc, that is not based on a protein scaffold but on an amphipathic SMA copolymer (Knowles et al., 2009; Orwick et al., 2012; Dorr et al., 2016; Lee et al., 2016). The SMA polymer assembles into $12 \mathrm{~nm}$ diameter discs and has been used for bR refolding and characterization (Orwick-Rydmark et al., 2012) as well as for native membrane solubilization of mitochondrial respiratory proteins (Long et al., 2013) and KcsA channel (Dorr et al., 2014). For the latter, the lipodiscs were formed by directly extracting native membrane fragments from the cells over-expressing the MP of interest. Therefore, this method has not only the potential to circumvent a detergent solubilization step, but can also generate homogenous soluble particles of the native membrane (Lee et al., 2016). Although the use of SMA lipodiscs for structure determination of MPs by NMR has not yet been demonstrated, they have been used for negative stain electron microscopy, yielding structures at a modest resolution $(>15 \AA)$ in a short timeframe (Postis et al., 2015). The slightly increased size in respect to MSP1D1-based NDs as well as potential heterogeneity of the native membrane fragments may on the one hand challenge NMR investigations but on the other hand also provide exciting new possibilities to study MPs in their native environment. In general, solution as well as solidstate NMR techniques may be considered when structural studies of MPs in SMA lipodiscs are intended.

\section{NMR applications}

NMR spectroscopy has the intrinsic capability to determine the three-dimensional structure of membrane proteins and to describe their local and global motions in a lipid bilayer nanodiscs system. To date, several MPs were already successfully reconstituted into nanodiscs and studied by solution NMR, including Aam-I (Lyukmanova et al., 2008), the helical transmembrane domains of CD4 (Gluck et al., 2009), KcsA (Shenkarev et al., 2009; Imai et al., 2012), VDAC-1 (Raschle et al., 2009), KvAP (Shenkarev et al., 2010b), the Pf1 coat protein, the viral protein p7, and the G-protein-coupled receptor CXCR1 (Park et al., 2011), VDAC-2 (Yu et al., 2012), bacteriorhodopsin (Etzkorn et al., 2013), OmpX (Hagn et al., 2013; Bibow et al., 2014), Integrin $\alpha_{\mathrm{IIb}}$ (Puthenveetil and Vinogradova, 2013), YgaP (Tzitzilonis et al., 2013), OmpA (Susac et al., 2014), Opa 60 (Fox et al., 2014), $\mu$-opioid receptor (Okude et al., 2015), BamA (Morgado et al., 2015), STIM1-TM (Wang et al., 2015) and Cytb $b_{5}$-CytP450 complex (Zhang et al., 2016). In addition, solid-state NMR has been applied to study MPs embedded into nanodiscs including the human CYP3A4 (Kijac et al., 2007), proteorhodopsin (Mors et al., 2013) or the Yersinia pestis outer membrane protein Ail (Ding et al., 2015a).

While the nanodisc system offers unique potential for NMR-based structural studies (Denisov and Sligar, 2016; Liang and Tamm, 2016), it is also accompanied by several challenges. In addition to the above discussed complex sample preparation, even perfectly optimized NDs are approaching/crossing the size limit of conventional solution NMR techniques. A basic requirement is therefore the use of transverse relaxation optimized spectroscopy (TROSY)-based experiments (Salzmann et al., 1998; Guo et al., 2008). In addition, the usage of deuterium labeling is normally required to obtain detailed insights of MPs inside nanodiscs (Etzkorn et al., 2013; Kofuku et al., 2014). However, even when using these techniques, it has been shown that more complex three- or four-dimensional NMR experiments are impeded by the fast relaxation mechanisms that result from the large rotational correlation times of the nanodisc particles. It has been shown for bR and OmpX in MSP1D1 nanodiscs (Etzkorn et al., 2013; Hagn et al., 2013) that while nearly all expected peaks are present in $2 \mathrm{D}{ }^{1} \mathrm{H}^{-15} \mathrm{~N}-\mathrm{HSQC}$ spectra and 3D HNCO spectra, no peaks of transmembrane protein regions could be detected in experiments that include $\mathrm{a}^{13} \mathrm{C}-{ }^{13} \mathrm{C}$ INEPT based magnetization transfer. In these cases, combinatorial isotope labeling can be used to allow (partial) resonance assignment (Etzkorn et al., 2013). Alternatively, NMRoptimized, smaller MSP constructs (see Section 'Choosing the MSP construct') can be used to sufficiently increase molecular tumbling and allow the usage of regular ${ }^{13} \mathrm{C}-{ }^{13} \mathrm{C}$ transfer sequences (Hagn et al., 2013). It has also been successfully shown that TOCSY-based ${ }^{13} \mathrm{C}-{ }^{13} \mathrm{C}$ transfer, combined with ${ }^{13} \mathrm{C}$ detection, can be used to study the transmembrane region of OmpX in MSDP1D1 NDs (Viegas et al., 2016). Remarkably, when using unified time-optimized interleaved acquisition (UTOPIA) setups this information can be obtained for free during the acquisition of a 3D NOESY-TROSY experiment (Viegas et al., 2016).

In general, it has been shown for various systems that nanodiscs can largely increase thermal stability of the inserted membrane protein in comparison to detergentbased environments (Etzkorn et al., 2013; Rues et al., 
2016). This feature can and should be exploited by using increased temperatures and/or prolonged experimental times when recording solution NMR experiments. In this respect, the increased stability can partly compensate for the increased molecular weight of NDs compared to micelles. In our experience, the spectral quality of MPs in nanodiscs considerably increases when measuring above $35^{\circ} \mathrm{C}$ (note that in this case also the phase transition temperature of the lipids should be considered, also see Section 'Removing detergent').

\section{Structure determination}

One of the greatest potentials of using NMR spectroscopy to study MPs incorporated into nanodiscs is the possibility of determining their 3D structure in solution, in a closeto-native and active conformation. Although the molecular weight of the MP-ND complex is high, it should still be under $200 \mathrm{kDa}$ and thus suitable for being studied by modern NMR techniques (Shenkarev et al., 2010a; Klammt et al., 2012; Opella 2013; Viegas et al., 2016). The first MP structure determined by NMR and using the ND technology was of the 180-residues bacterial outer-membrane protein OmpX (PDB code: 2M06) (Hagn et al., 2013). In their work, the authors developed a short MSP (MSP1D1 $\Delta$ H5) variant which resulted in smaller size NDs. The use of these NDs, allied with highly deuterated protein and lipids provided significant improvements for the structure determination process. Although the obtained structure revealed the same topology for the transmembrane part as previously shown by NMR (Fernandez et al., 2004) and X-ray crystallography (Vogt and Schulz, 1999), distinct differences were found for the extracellular extensions of the $\beta$-sheets. These differences were attributed to the immobilization of these segments due to the crystal packing and the cryogenic temperatures used for data collection. The constraining of the loops was not verified when using NDs and this result was in agreement with NMR and MD data. By determining the structure of OmpX in detergents but using otherwise identical buffer conditions as for the NDs, it was further shown that, when compared to the nanodisc structure, each strand was up to two residues shorter, giving a good example that the membrane mimetic can have a significant impact on the determined structure. The difference was attributed to the slightly denaturing effect of the detergent at the water boundary and differences in hydrophobic coverage.

The structure of OmpX in NDs was later refined using residual dipolar couplings (RDCs) (Bibow et al., 2014). The authors obtained the RDCs with the OmpX protein embedded in nanodiscs (also using the MSP1D1 $\Delta \mathrm{H} 5$ variant) and using the Pf1 phage as alignment medium. The structure was calculated using the published experimental restraints (Hagn et al., 2013) together with the measured RDCs and showed and improved accuracy including, in particular, better-defined orientations of the $\mathrm{N}-\mathrm{H}$ bonds. This work demonstrated that membrane protein bond orientation in nanodiscs can be obtained by measuring RDCs via Pf1 phages as alignment medium.

Further refinement of the 3D structure of OmpX in NDs was performed using a NOE-based approach to assign selectively (methyl) labeled protein (Hagn and Wagner, 2015). In addition, RDCs were also obtained, using Pf1 phages as an alignment medium. By using the NOE information between the protonated head groups of the lipids and the side chain methyl groups and paramagnetic relaxation enhancement (PRE) experiments (obtained with $\mathrm{Gd}^{3+}$-modified lipids), the authors were also able to determine the position of OmpX in the phospholipid bilayer.

A similar approach, coupled with chemical shift perturbation (CSP) analysis and backbone relaxation measurements, was used to study the structural and dynamic properties of the interactions between the membrane and the GTPase domain of tethered Rheb (Mazhab-Jafari et al., 2013). This study provided the first experimental evidence for membrane-dependent regulation of the structure and function of a GTPase at atomic resolution.

In another example, a mixed approach, using restraints determined with solution NMR spectroscopy in detergent micelles and NDs in conjunction with MD simulations in a lipid bilayer was used to determine the structure of opacity-associated protein Opa ${ }_{60}$ (Fox et al., 2014). The final structural ensemble showed that the extracellular loops (which bind to the host receptors) interact with each other and are dynamic on the nanosecond time scale.

The conformation and ligand binding properties of the protein $\mathrm{Bcl}-\mathrm{x}_{\mathrm{L}}$ upon membrane integration was also studied by NMR, applying the nanodisc technology (Yao et al., 2015). Bcl- $\mathrm{x}_{\mathrm{L}}$ consists of a soluble domain and a membrane anchoring domain and all previous structures lacked the hydrophobic C-terminal membrane anchor, despite its potential importance for the protein function. In their work, the authors (Yao et al., 2015) tackled these issues by reconstituting the full-length Bcl- $\mathrm{x}_{\mathrm{L}}$ into NDs, macrodiscs and liposomes, which allowed a direct structural comparison of its various conformations. By comparing the ${ }^{1} \mathrm{H}-{ }^{15} \mathrm{~N}$-HSQC spectra acquired under different conditions, the authors showed that the soluble domain does not experience significant conformational changes upon membrane integration, contrary to some current 
models. Besides, it was shown that the membrane-integrated protein has higher affinity for a pro-apoptotic peptide than the truncated version, indicating that membrane integration alters the binding activity. In a later study (Yao et al., 2016), the authors used PRE data to show that, although the $\mathrm{C}$-terminal part of $\mathrm{Bcl}-\mathrm{x}_{\mathrm{L}}$ is inserted into the membrane, it still retains a high degree of conformational dynamics, which may facilitate its cleavage in aqueous solution.

\section{Dynamics}

In addition to the 3D structure, knowledge of the dynamic processes underlying conformational changes or ligand recognition are key aspects to fully understand MPs' functional mechanisms in a native environment and therefore often the focus of NMR-based studies.

One example is given by the solution NMR investigation of the insertase BamA in three different membrane mimetics: LDAO micelles, DMPC: $\mathrm{DiC}_{7}-\mathrm{PC}$ bicelles and MSP1D1:DMPC nanodiscs (Morgado et al., 2015). Among their findings, the authors observed distinct time scales of dynamic behavior for the BamA $\beta$-barrel and its extracellular 'lid loop' L6, which is essential for BamA function (Ni et al., 2014). The results helped to clarify why parts of this loop have poor electron densities in BamA crystal structures (Ni et al., 2014) and provided an important piece of information towards understanding the functional mechanism of the BamA insertase at the atomic level.

Another example is the investigation of the dynamics of the transmembrane and intracellular domains of p75NTR (a type I integral membrane protein) in NDs of various sizes and compositions (Mineev et al., 2015). The p75NTR has an extracellular domain (ECD), a helical transmembrane domain (TMD) and an intracellular domain, which, in turn, can be divided into three parts: the chopper domain and a linker region. Despite the availability of a proposed mechanism for the activity of the p75NTR receptor (Vilar et al., 2009), the role of the chopper domain in the activation of the receptor or the connection between the conformations of the TMDs and ECDs and the state of the intracellular part remain poorly understood. By measuring longitudinal $\left(T_{1}\right)$ and transverse $\left(T_{2}\right)$ relaxation times, heteronuclear ${ }^{1} \mathrm{H}^{-15} \mathrm{~N} \mathrm{NOE}$, and cross-correlated relaxation rates of different constructs, the authors concluded that the motions of the death domain are uncoupled from the TM helix. Together with the structural data acquired, the results indicate that the previously proposed mechanism does fit in vivo data well but contradicts the structural information, therefore pointing the need to consider alternative mechanisms.

A similar approach was applied to the study of the protein synaptobrevin (composed by an ectodomain, containing a SNARE motif, and a transmembrane domain) inserted into NDs (Brewer et al., 2011). In this study the authors found that most of the residues belonging to the SNARE motif are unstructured and flexible in the presence of NDs, as opposed to what happens in the presence of DPC micelles. This leaves the SNARE motif accessible for SNARE complex formation and shows that NDs are a much better membrane mimetic than micelles.

\section{Interaction studies}

Nanodiscs can be an ideal tool to study the functional properties of integral membrane proteins in a lipid environment, under defined conditions (Inagaki et al., 2013). They can allow the control of the oligomerization state of a given MP (also see Section 'Choosing the MSP-to-lipid and MSP-to-MP ratios') and offer high-precision control of lipid content (Lee et al., 2015), thus enabling the study of specific effects of different lipid environments. Unlike proteoliposomes, both the intra- and extracellular sides of the protein are directly accessible, ensuring homogenously accessible binding sites and allowing the investigation of signaling events that require binding events on both sides.

It is generally accepted that NDs offer good properties to examine lipid-protein interactions (Schuler et al., 2013). Still, when probing the interaction of protein/peptides with NDs, it should be tested whether the interaction partner unspecifically interacts with the MSP. Although, this could be the case with soluble MSP, it may no longer be true when nanodiscs have been formed. Upon formation of the NDs, the hydrophobic parts of MSP are not directly accessible anymore. Therefore, when testing whether an interaction partner unspecifically interacts with MSP, it is advised to not use soluble MSP but MSP in its nanodisc conformation with neutral lipids. If NDs with only one kind of lipids (e.g. DMPC) do not show any interaction while NDs with different lipids do interact, unspecific MSP interaction can be largely excluded.

One illustration of the power of the combination between NMR and the nanodisc technology applied to the investigation of protein interactions is given by the study of the interaction between the phosphoinositide (PI)binding effector protein [FYVE domain of early endosome antigen 1 (EEA1) - EEA1 FYVE] and a PI incorporated into 
nanodiscs [PI(3)P] (Kobashigawa et al., 2011; Yokogawa et al., 2012). Although models of EEA1 FYVE bound to a PI(3)P-embedded membrane existed (based on structural analyses using soluble PI(3)P and the NMR data in a micelle system], the relative orientations of EEA1 FYVE to the membrane were not consistent in these two studies. Moreover, no experimental data had been reported to validate the binding mode of EEA1 FYVE with PI(3)P in the lipid bilayer system. In their study, the authors used CSP, transferred cross-saturation (TCS) and PRE experiments to map the residues of EEA1 FYVE involved in recognition of PI(3)P and to estimate a $K_{\mathrm{D}}$ value of about 105 $\mu \mathrm{M}$. Besides, CSP experiments only in the presence of empty nanodiscs allowed to identify at least one residue involved in the interaction with the membrane. The TCS experiments determined the direct binding surface on EEA1 FYVE to PI(3)P incorporated membrane, and the orientation to the membrane was confirmed by the PRE experiments.

The interaction of the oncogenic protein K-Ras4B tethered to nanodiscs with effector protein RAS-binding domains was also studied by solution-state NMR spectroscopy (Mazhab-Jafari et al., 2015). Using selective ${ }^{13} \mathrm{C}$-labeling in combination with molecular docking, the authors were able to gather important information on the orientation of the protein with respect to the lipid bilayer. Besides, the data also allowed to identify particular protein-lipid membrane interactions involved in molecular recognition and provided information about the dynamics of small-molecule-binding sites at the protein-lipid interface.

In another study, the interaction between the CCchemokine receptor 5 (CCR5) reconstituted into nanodiscs and its ligand (MIP-1 $\alpha$ ) was investigated (Yoshiura et al., 2010). Due to the receptor's instability in detergents, structural studies have been difficult. Reconstitution of CCR 5 in nanodiscs allowed to maintain its function for more than $24 \mathrm{~h}$, thus enabling structural analysis by solution NMR. Methyl-directed TCS experiments allowed to identify the residues of MIP-1 $\alpha$ in close proximity of the receptor in the complex. The TCS data suggests that the protective influence on HIV-1 infection of a single nucleotide polymorphism of MIP- $1 \alpha$ is due to its change in the interaction mode between MIP-1 $\alpha$ and CCR5. The same group also studied the effect of different mimetic environments on the exchange rates for the conformational equilibrium of $\beta_{2}$-adrenergic receptor upon binding to various ligands. They found that the exchange rates were significantly different in detergents and nanodiscs. These fast timescales of GPCR signaling permit the rapid neurotransmission and sensory perception (Kofuku et al., 2014).
Another example is the study of the interaction of granuphilin-C2A domain with phosphatidylinositol 4, 5-bisphosphate $\left[\mathrm{PI}(4,5) \mathrm{P}_{2}\right]$ head group and $\mathrm{PI}(4$, 5) $\mathrm{P}_{2}$-nanodisc (Wan et al., 2015). NMR-based titration experiments proved the interaction and allowed to identify the binding site of granuphilin-C2A. These results were further confirmed by molecular docking, mutation and isothermal titration calorimetry (ITC) analysis and demonstrated that $\mathrm{PI}(4,5) \mathrm{P}_{2}$ binds not only to the concave surface of granuphilin-C2A domain but also that strand $\beta 5$ is affected. The data provided a better understanding of the role of granuphilin-C2A domain in regulating exocytosis.

An important topic to address when studying MP-lipid interactions is the role the lipids themselves may have in the overall function of the protein (e.g. signal transduction). MP-lipid interactions may have an effect on the physical properties of the lipid bilayer and the behavior of its components. Besides interacting with the inserted proteins, lipid head groups may also interact with ions that influence the protein's function. In this sense, several NMR-based studies (Shenkarev et al., 2009, 2014; Boettcher et al., 2011; Zhang et al., 2014) have been performed. For instance, solid-state NMR has been applied to study the influence of $\mathrm{Ca}^{2+}$ on the structure and dynamics of lipid bilayers (Boettcher et al., 2011). Using the ${ }^{1} \mathrm{H}-{ }^{-1} \mathrm{H},{ }^{13} \mathrm{C}-{ }^{13} \mathrm{C}$, and ${ }^{13} \mathrm{C}{ }^{31} \mathrm{P}$ dipolar interactions, distances up to $\sim 6 \AA$ could be measured between atoms and by combining this with MD simulations the authors showed a $\mathrm{Ca}^{2+}$-induced conformational change and clustering of lipid head groups in POPC/POPS NDs. Because ${ }^{31} \mathrm{P}$ chemical shift anisotropy is sensitive to alterations in the orientation and motion of lipid head groups, it can be used as a probe for studying MP-lipid interactions. This approach was used to probe the change in the chemical environment of phospholipids in response to $\mathrm{Ca}^{2+}$ addition (Zhang et al., 2014). A similar spectrum was observed upon $\alpha$-synuclein binding, indicating that $\mathrm{Ca}^{2+}$ and $\alpha$-synuclein may interact with the same region in the lipid.

\section{Limitations}

Even if NDs are a very good model system for mimicking natural membranes, their nanoscale size and the presence of a scaffold protein still introduce a distinct environment. This can affect main physical parameters characterizing bilayers, including the temperature of main phase transition, the shape, the curvature, and the lateral pressure, which can be studied using SAXS, AFM, EM, NMR and/ 
or fluorescence techniques. In general, the phase transitions are broadened and shifted to higher temperatures and it was found that the so-called boundary lipids do not melt at the main phase transition temperature but are further stabilized in the gel phase by their interaction with the scaffold (Shaw et al., 2004; Denisov et al., 2005; Kijac et al., 2010). This lack of cooperativity of a larger proportion of the lipids is, however, thought to mimic the natural membranes quite well, which often also comprises considerable protein crowding. Nevertheless, the fixed geometry due to the MSP can generally introduce a higher lateral pressure to the bilayer. Additionally, not all lipids may be suitable for ND formation and depending on the type of lipid used and their chain lengths, hydrophobic mismatch between the lipids and the scaffold may appear, leading to non-flat but curved surfaces and/or non-homogenous thickness (Denisov et al., 2005; Skar-Gislinge et al., 2010).

It may also not be clear for all proteins whether they insert in the center of the disc and are surrounded by lipids, or if unspecific interactions may favor nanodisc assembly with direct MP-MSP contacts. NMR studies have however shown that, at least for the OmpX protein, spectral quality will be compromised if not even one layer of lipids is around the inserted MP (Hagn et al., 2013). This observation suggests that, if given the option, OmpX preferentially resides in the center of the discs surrounded by lipids.

While in general NDs with different total amounts of charged lipids can be easily produced, a charge gradient between the two sides of the membrane (as found for several cellular membranes) cannot be generated using conventional self-assembly reactions. However, in theory, it may be possible to incorporate MPs into differentially charged bilayers by, for instance, extracting native cellular membranes. In respect to the experimental characterization of the inserted MP, a substantial extinction as well as the high helical propensity of the MSP render some techniques difficult such as circular dichroism and the concentration determination using the absorbance at $280 \mathrm{~nm}$ wavelength. The latter can interfere with the determination of the average number of inserted proteins per disc and may be partially overcome using quantitative SDS-PAGE, MS, or SEC under denaturing conditions.

\section{Perspectives/conclusion}

As outlined above, using NDs may be demanding in terms of sample preparation and NMR data acquisition. Therefore, one should consider whether the study of the target MP, in particular also in respect to the scientific question that should be addressed, really benefits from the ND environment. It has been proposed to use the ND environment as a reference for the native MP state and, in cases where NMR spectra in detergent micelles are similar (indicative of similar structures), to proceed with a more detailed structural characterization in the detergent system (Shenkarev et al., 2010a; Tzitzilonis et al., 2013; Susac et al., 2014).

Nevertheless, in addition to providing a near native local environment, there are unique properties of the nanodisc system with a great potential for NMR-based studies. These properties include in particular the clear definition and kinetic stability of the assembled NDs, meaning that molecular exchange processes are very slow and once formed the lipid composition, disc size and number of inserted MPs should not vary considerably over time. In addition to the absence of detergents, these features may not be given for other membrane mimetic systems such as bicelles. Figure 5 gives an overview of selected useful properties of the NDs for NMR-based studies. For instance, as discussed above, it has been shown that NDs, unlike micelles, can be used to measure RDCs using established alignment media (Bibow et al., 2014; Hagn and Wagner, 2015) (Figure 5A). This property may offer rather straightforward means to check the topology of the target MP. In addition, it has been shown that large nanodiscs (i.e. macrodiscs) align themselves in magnetic field and can therefore be used as alignment media (Park et al., 2011).

Due to the good definition of and flexibility in the lipid content of NDs, they offer an ideal platform to study peptide or protein-membrane interactions. It has already been shown for $\alpha$-synuclein that residue specific information regarding membrane association can be obtained with the combination of ND and NMR (Zhang et al., 2014). By varying the lipid content of the NDs, one could study the effects of different lipid properties on the peptidemembrane interaction (Figure 5B).

In principle, the ND system could also be used to not only study MP-MP interactions within the same membrane, but also MP-MP interactions of proteins embedded in two separate membranes as present in viral docking and/or membrane fusion (Figure $5 \mathrm{C}$ ). In this respect the scaffold protein could prevent the actual fusion step, thus trapping the initial stages of the process.

The good solvent accessibility of the embedded MP can also be exploited in various scenarios. In addition to the (simultaneous) binding of interaction partners from both sides, this property could be useful to generate a rather homogenous distribution of biradicals for dynamic nuclear polarization (DNP) experiments of MPs, which 
A

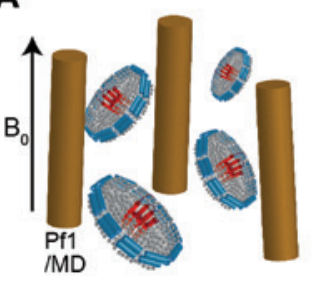

B

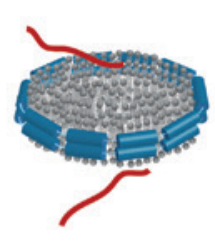

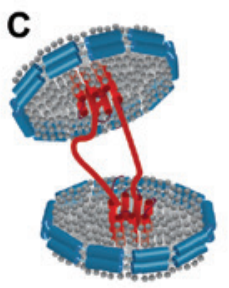

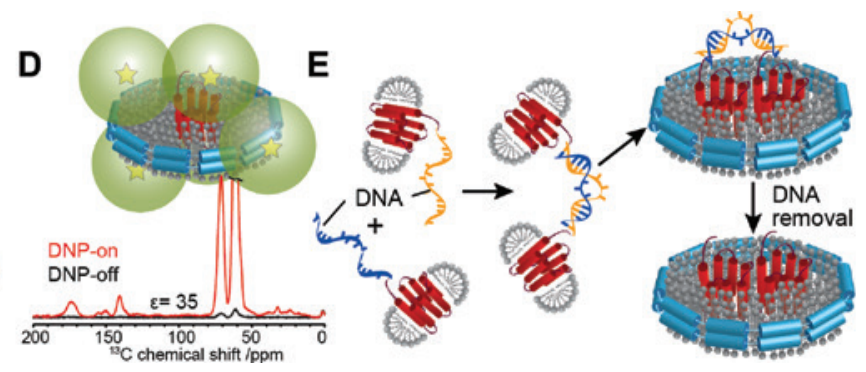

Figure 5: Selected useful properties and future potential of NDs for NMR-based studies.

(A) NDs can be used to measure RDCs using established alignment media (Bibow et al., 2014; Hagn and Wagner, 2015). (B) The good definition of and flexibility in the lipid content of NDs provides a suitable platform for studying lipid-protein interactions (Zhang et al., 2014). (C) the ND system offers the potential to probe interactions between protein embedded into separate NDs. (D) The good solvent accessibility of NDs can be used to generate a homogenous distribution of biradicals for dynamic nuclear polarization (DNP) experiments. Decent enhancement factors are found for bR in MSP1D1 nanodiscs, which enables characterization of low amounts ( $38 \mu \mathrm{g})$ of bR (unpublished data). (E) ND systems offer the unique possibility to insert a defined (small) number of membrane proteins into one disc using, for instance, a DNA origami approach (Raschle et al., 2015) allowing the study of MP-MP interactions within the same bilayer (Figure 5E adapted with permission from Raschle et al., 2015. @2015 American Chemical Society.)

are so far normally carried out in proteoliposomes that could introduce a radical gradient between the inside and outside of the liposome. Our initial DNP measurements confirm that the ND environment is well accessible to DNP techniques (Figure 5D, unpublished data).

It is noteworthy that the ND systems provides unique possibility to insert a defined (small) number of membrane proteins into one disc. Using an DNA origami approach, it was shown that either two or three MPs can be inserted in a well-defined way into one single ND (Figure 5E) (Raschle et al., 2015). While DNA design and upscaling may be demanding, this approach combined with the 'exchangestability' of the ND system, can produce extraordinary oligomer homogeneity offering promising potential for studies of MP-MP interaction within the same bilayer.

Although the work with nanodiscs may be tedious, it should be pointed out that solution NMR studies of larger MPs in nanodiscs (up to a size of MSP1D1) are well suitable. This means that bad NMR spectra obtained on these systems are not a result of the total particles size but can either be attributed to not optimal sample preparation or intrinsic features of the MP. In this respect, if sample preparation is 'optimal' (e.g. according to the SEC profile and functional assays) the absence of decent NMR spectra may already be attributed to intrinsic properties of the MP, such as dynamic or heterogeneous conformations.

All in all, due to the intrinsic potential of the system paired with the recent advances in the field, it can be anticipated that nanodiscs will become a standard tool in the NMR-based studies of membrane proteins. In this respect it may offer a platform to obtain site-resolved insights into structure, function, dynamics and/or interactions of membrane associated processes in a near native environment.

Acknowledgments: The authors acknowledge access to the Jülich-Düsseldorf Biomolecular NMR Center, support by Dr. Fabien Aussenac (Bruker Biospin) and thank the DFG for support through the Emmy Noether grants ET103/2-1 to M.E. as well as the Marie Sklodowska-Curie Grant No. 660258 to A.V. T.V. acknowledges support from the International NRW Research School iGRASPseed.

\section{References}

Ajees, A.A., Anantharamaiah, G.M., Mishra, V.K., Hussain, M.M., and Murthy, H.M. (2006). Crystal structure of human apolipoprotein A-I: insights into its protective effect against cardiovascular diseases. Proc. Natl. Acad. Sci. USA 103, 2126-2131.

Alami, M., Dalal, K., Lelj-Garolla, B., Sligar, S.G., and Duong, F. (2007). Nanodiscs unravel the interaction between the SecYEG channel and its cytosolic partner SecA. EMBO J. 26, 1995-2004.

Anantharamaiah, G.M., Jones, J.L., Brouillette, C.G., Schmidt, C.F., Chung, B.H., Hughes, T.A., Bhown, A.S., and Segrest, J.P. (1985). Studies of synthetic peptide analogs of the amphipathic helix. Structure of complexes with dimyristoyl phosphatidylcholine. J. Biol. Chem. 260, 10248-10255.

Baas, B.J., Denisov, I.G., and Sligar, S.G. (2004). Homotropic cooperativity of monomeric cytochrome P450 3A4 in a nanoscale native bilayer environment. Arch. Biochem. Biophys. 430, 218-228.

Baker, S.E., Hopkins, R.C., Blanchette, C.D., Walsworth, V.L., Sumbad, R., Fischer, N.O., Kuhn, E.A., Coleman, M., 
Chromy, B.A., Letant, S.E., et al. (2009). Hydrogen production by a hyperthermophilic membrane-bound hydrogenase in water-soluble nanolipoprotein particles. J. Am. Chem. Soc. 131, 7508-7509.

Banerjee, S., Huber, T., and Sakmar, T.P. (2008). Rapid incorporation of functional rhodopsin into nanoscale apolipoprotein bound bilayer (NABB) particles. J. Mol. Biol. 377, 1067-1081.

Bao, H., Goldschen-Ohm, M., Jeggle, P., Chanda, B., Edwardson, J.M., and Chapman, E.R. (2016). Exocytotic fusion pores are composed of both lipids and proteins. Nat. Struct. Mol. Biol. 23, 67-73.

Bayburt, T.H. and Sligar, S.G. (2003). Self-assembly of single integral membrane proteins into soluble nanoscale phospholipid bilayers. Protein Sci. 12, 2476-2481.

Bayburt, T.H. and Sligar, S.G. (2010). Membrane protein assembly into Nanodiscs. FEBS Lett. 584, 1721-1727.

Bayburt, T.H., Carlson, J.W., and Sligar, S.G. (1998). Reconstitution and imaging of a membrane protein in a nanometer-size phospholipid bilayer. J. Struct. Biol. 123, 37-44.

Bayburt, T.H., Grinkova, Y.V., and Sligar, S.G. (2002). Self-assembly of discoidal phospholipid bilayer nanoparticles with membrane scaffold proteins. Nano Lett. 2, 853-856.

Bayburt, T.H., Grinkova, Y.V., and Sligar, S.G. (2006). Assembly of single bacteriorhodopsin trimers in bilayer nanodiscs. Arch. Biochem. Biophys. 450, 215-222.

Bayburt, T.H., Leitz, A.J., Xie, G., Oprian, D.D., and Sligar, S.G. (2007). Transducin activation by nanoscale lipid bilayers containing one and two rhodopsins. J. Biol. Chem. 282, 14875-14881.

Bhat, S., Sorci-Thomas, M.G., Tuladhar, R., Samuel, M.P., and Thomas, M.J. (2007). Conformational adaptation of apolipoprotein A-I to discretely sized phospholipid complexes. Biochemistry $46,7811-7821$.

Bibow, S., Carneiro, M.G., Sabo, T.M., Schwiegk, C., Becker, S., Riek, R., and Lee, D. (2014). Measuring membrane protein bond orientations in nanodiscs via residual dipolar couplings. Protein Sci. 23, 851-856.

Bocquet, N., Kohler, J., Hug, M.N., Kusznir, E.A., Rufer, A.C., Dawson, R.J., Hennig, M., Ruf, A., Huber, W., and Huber, S. (2015). Real-time monitoring of binding events on a thermostabilized human A2A receptor embedded in a lipid bilayer by surface plasmon resonance. Biochim. Biophys. Acta 1848 , 1224-1233.

Boettcher, J.M., Davis-Harrison, R.L., Clay, M.C., Nieuwkoop, A.J., Ohkubo, Y.Z., Tajkhorshid, E., Morrissey, J.H., and Rienstra, C.M. (2011). Atomic view of calcium-induced clustering of phosphatidylserine in mixed lipid bilayers. Biochemistry 50 , 2264-2273.

Boldog, T., Li, M., and Hazelbauer, G.L. (2007) Using nanodiscs to create water-soluble transmembrane chemoreceptors inserted in lipid bilayers. In: Methods Enzymol., Vol. 423, Melvin, I. Simon, Crane, Alexandrine, eds. (New York: Elsevier Inc.), pp. 317-335.

Borch, J. and Hamann, T. (2009). The nanodisc: a novel tool for membrane protein studies. Biol. Chem. 390, 805-814.

Borch, J., Torta, F., Sligar, S.G., and Roepstorff, P. (2008). Nanodiscs for immobilization of lipid bilayers and membrane receptors: kinetic analysis of cholera toxin binding to a glycolipid receptor. Anal. Chem. 80, 6245-6252.

Borhani, D.W., Rogers, D.P., Engler, J.A., and Brouillette, C.G. (1997). Crystal structure of truncated human apolipoprotein A-I suggests a lipid-bound conformation. Proc. Natl. Acad. Sci. USA 94, 12291-12296.

Brewer, K.D., Li, W., Horne, B.E., and Rizo, J. (2011). Reluctance to membrane binding enables accessibility of the synaptobrevin SNARE motif for SNARE complex formation. Proc. Natl. Acad. Sci. USA 108, 12723-12728.

Brouillette, C.G. and Anantharamaiah, G.M. (1995). Structural models of human apolipoprotein A-I. Biochim. Biophys. Acta 1256, 103-129.

Brouillette, C.G., Anantharamaiah, G.M., Engler, J.A., and Borhani, D.W. (2001). Structural models of human apolipoprotein A-I: a critical analysis and review. Biochim. Biophys. Acta 1531, 4-46.

Cappuccio, J.A., Blanchette, C.D., Sulchek, T.A., Arroyo, E.S., Kralj, J.M., Hinz, A.K., Kuhn, E.A., Chromy, B.A., Segelke, B.W., Rothschild, K.J., et al. (2008). Cell-free co-expression of functional membrane proteins and apolipoprotein, forming soluble nanolipoprotein particles. Mol. Cell. Proteomics 7, 2246-2253.

Catoire, L.J., Warnet, X.L., and Warschawski, D.E. (2014). Micelles, bicelles, amphipols, nanodiscs, liposomes, or intact cells: the hitchhiker's guide to the study of membrane proteins by NMR. In: Membrane Proteins Production for Structural Analysis. Isabelle Mus-Veteau, ed. (New York: Springer), pp. 315-345.

Chromy, B.A., Arroyo, E., Blanchette, C.D., Bench, G., Benner, H., Cappuccio, J.A., Coleman, M.A., Henderson, P.T., Hinz, A.K., Kuhn, E.A., et al. (2007). Different apolipoproteins impact nanolipoprotein particle formation. J. Am. Chem. Soc. 129, 14348-14354.

Civjan, N.R., Bayburt, T.H., Schuler, M.A., and Sligar, S.G. (2003). Direct solubilization of heterologously expressed membrane proteins by incorporation into nanoscale lipid bilayers. BioTechniques 35, 556-560, 562-563.

D’Antona, A.M., Xie, G., Sligar, S.G., and Oprian, D.D. (2014). Assembly of an activated rhodopsin-transducin complex in nanoscale lipid bilayers. Biochemistry 53, 127-134.

Dalal, K., Nguyen, N., Alami, M., Tan, J., Moraes, T.F., Lee, W.C., Maurus, R., Sligar, S.S., Brayer, G.D., and Duong, F. (2009). Structure, binding, and activity of Syd, a SecY-interacting protein. J. Biol. Chem. 284, 7897-7902.

Denisov, I.G. and Sligar, S.G. (2016). Nanodiscs for structural and functional studies of membrane proteins. Nat Struct Mol Biol 23, 481-486.

Denisov, I.G., Grinkova, Y.V., Lazarides, A.A., and Sligar, S.G. (2004). Directed self-assembly of monodisperse phospholipid bilayer nanodiscs with controlled size. J. Am. Chem. Soc. 126, 3477-3487.

Denisov, I.G., McLean, M.A., Shaw, A.W., Grinkova, Y.V., and Sligar, S.G. (2005). Thermotropic phase transition in soluble nanoscale lipid bilayers. J. Phys. Chem. B 109, 15580-15588.

Ding, Y., Yao, Y., and Marassi, F.M. (2013). Membrane protein structure determination in membrana. Acc. Chem. Res. 46, 2182-2190.

Ding, Y., Fujimoto, L.M., Yao, Y., and Marassi, F.M. (2015a). Solidstate NMR of the Yersinia pestis outer membrane protein Ail in lipid bilayer nanodiscs sedimented by ultracentrifugation. J. Biomol. NMR 61, 275-286.

Ding, Y., Fujimoto, L.M., Yao, Y., Plano, G.V., and Marassi, F.M. (2015b). Influence of the lipid membrane environment on structure and activity of the outer membrane protein Ail from Yersinia pestis. Biochim. Biophys. Acta 1848, 712-720. 
Dorr, J.M., Koorengevel, M.C., Schafer, M., Prokofyev, A.V., Scheidelaar, S., van der Cruijsen, E.A., Dafforn, T.R., Baldus, M., and Killian, J.A. (2014). Detergent-free isolation, characterization, and functional reconstitution of a tetrameric $\mathrm{K}^{+}$channel: the power of native nanodiscs. Proc. Natl. Acad. Sci. USA 111, 18607-18612.

Dorr, J.M., Scheidelaar, S., Koorengevel, M.C., Dominguez, J.J., Schafer, M., van Walree, C.A., and Killian, J.A. (2016). The styrene-maleic acid copolymer: a versatile tool in membrane research. Eur. Biophys. J. 45, 3-21.

Duan, H., Civjan, N.R., Sligar, S.G., and Schuler, M.A. (2004). Co-incorporation of heterologously expressed Arabidopsis cytochrome $\mathrm{P} 450$ and $\mathrm{P} 450$ reductase into soluble nanoscale lipid bilayers. Arch. Biochem. Biophys. 424, 141-153.

Eggensperger, S., Fisette, O., Parcej, D., Schafer, L.V., and Tampe, R. (2014). An annular lipid belt is essential for allosteric coupling and viral inhibition of the antigen translocation complex TAP (transporter associated with antigen processing). J. Biol. Chem. 289, 33098-33108.

Etzkorn, M., Raschle, T., Hagn, F., Gelev, V., Rice, A.J., Walz, T., and Wagner, G. (2013). Cell-free expressed bacteriorhodopsin in different soluble membrane mimetics: biophysical properties and NMR accessibility. Structure 21, 394-401.

Fernandez, C., Hilty, C., Wider, G., Guntert, P., and Wuthrich, K. (2004). NMR structure of the integral membrane protein OmpX. J. Mol. Biol. 336, 1211-1221.

Fox, D.A., Larsson, P., Lo, R.H., Kroncke, B.M., Kasson, P.M., and Columbus, L. (2014). Structure of the Neisserial outer membrane protein $\mathrm{Opa}_{60}$ : loop flexibility essential to receptor recognition and bacterial engulfment. J. Am. Chem. Soc. 136, 9938-9946.

Frauenfeld, J., Gumbart, J., Sluis, E.O., Funes, S., Gartmann, M., Beatrix, B., Mielke, T., Berninghausen, O., Becker, T., Schulten, K., et al. (2011). Cryo-EM structure of the ribosome-SecYE complex in the membrane environment. Nat. Struct. Mol. Biol. $18,614-621$.

Frauenfeld, J., Loving, R., Armache, J.P., Sonnen, A.F., Guettou, F., Moberg, P., Zhu, L., Jegerschold, C., Flayhan, A., Briggs, J.A., et al. (2016). A saposin-lipoprotein nanoparticle system for membrane proteins. Nat. Methods 13, 345-351.

Gao, T., Petrlova, J., He, W., Huser, T., Kudlick, W., Voss, J., and Coleman, M.A. (2012). Characterization of de novo synthesized GPCRs supported in nanolipoprotein discs. PLoS One 7, e44911.

Gao, Y., Cao, E., Julius, D., and Cheng, Y. (2016). TRPV1 structures in nanodiscs reveal mechanisms of ligand and lipid action. Nature 534, 347-351.

Gluck, J.M., Wittlich, M., Feuerstein, S., Hoffmann, S., Willbold, D., and Koenig, B.W. (2009). Integral membrane proteins in nanodiscs can be studied by solution NMR spectroscopy. J. Am. Chem. Soc. 131, 12060-12061.

Grinkova, Y.V., Denisov, I.G., and Sligar, S.G. (2010). Engineering extended membrane scaffold proteins for self-assembly of soluble nanoscale lipid bilayers. Protein Eng. Des. Sel. 23, 843-848.

Guo, C., Zhang, D., and Tugarinov, V. (2008). An NMR experiment for simultaneous TROSY-based detection of amide and methyl groups in large proteins. J. Am. Chem. Soc. 130, 10872-10873.

Hagn, F. and Wagner, G. (2015). Structure refinement and membrane positioning of selectively labeled $\mathrm{OmpX}$ in phospholipid nanodiscs. J. Biomol. NMR 61, 249-260.
Hagn, F., Etzkorn, M., Raschle, T., and Wagner, G. (2013). Optimized phospholipid bilayer nanodiscs facilitate high-resolution structure determination of membrane proteins. J. Am. Chem. Soc. 135, 1919-1925.

Hartley, M.D., Schneggenburger, P.E., and Imperiali, B. (2013). Lipid bilayer nanodisc platform for investigating polyprenol-dependent enzyme interactions and activities. Proc. Natl. Acad. Sci. USA 110, 20863-20870.

Hiller, S. and Wagner, G. (2012) Solution NMR spectroscopy of integral membrane proteins. In: Comprehensive Biophysics, Vol 5, Egelman, Tamm. eds. (Burlington: Elsevier Inc.), pp 120-138.

Imai, S., Osawa, M., Mita, K., Toyonaga, S., Machiyama, A., Ueda, T., Takeuchi, K., Oiki, S., and Shimada, I. (2012). Functional equilibrium of the KcsA structure revealed by NMR. J. Biol. Chem. 287, 39634-39641.

Inagaki, S., Ghirlando, R., White, J.F., Gvozdenovic-Jeremic, J., Northup, J.K., and Grisshammer, R. (2012). Modulation of the interaction between neurotensin receptor NTS1 and $\mathrm{G}_{\mathrm{q}}$ protein by lipid. J. Mol. Biol. 417, 95-111.

Inagaki, S., Ghirlando, R., and Grisshammer, R. (2013). Biophysical characterization of membrane proteins in nanodiscs. Methods 59, 287-300.

Katayama, H., Wang, J., Tama, F., Chollet, L., Gogol, E.P., Collier, R.J., and Fisher, M.T. (2010). Three-dimensional structure of the anthrax toxin pore inserted into lipid nanodiscs and lipid vesicles. Proc. Natl. Acad. Sci. USA 107, 3453-3457.

Katzen, F., Fletcher, J.E., Yang, J.P., Kang, D., Peterson, T.C., Cappuccio, J.A., Blanchette, C.D., Sulchek, T., Chromy, B.A., Hoeprich, P.D., et al. (2008). Insertion of membrane proteins into discoidal membranes using a cell-free protein expression approach. J. Proteome Res. 7, 3535-3542.

Kawai, T., Caaveiro, J.M., Abe, R., Katagiri, T., and Tsumoto, K. (2011). Catalytic activity of MsbA reconstituted in nanodisc particles is modulated by remote interactions with the bilayer. FEBS Lett. 585, 3533-3537.

Kedrov, A., Sustarsic, M., de Keyzer, J., Caumanns, J.J., Wu, Z.C., and Driessen, A.J. (2013). Elucidating the native architecture of the YidC: ribosome complex. J. Mol. Biol. 425, 4112-4124.

Kijac, A.Z., Li, Y., Sligar, S.G., and Rienstra, C.M. (2007). Magicangle spinning solid-state NMR spectroscopy of nanodiscembedded human CYP3A4. Biochemistry 46, 13696-13703.

Kijac, A., Shih, A.Y., Nieuwkoop, A.J., Schulten, K., Sligar, S.G., and Rienstra, C.M. (2010). Lipid-protein correlations in nanoscale phospholipid bilayers determined by solid-state nuclear magnetic resonance. Biochemistry 49, 9190-9198.

Klammt, C., Maslennikov, I., Bayrhuber, M., Eichmann, C., Vajpai, N., Chiu, E.J., Blain, K.Y., Esquivies, L., Kwon, J.H., Balana, B., et al. (2012). Facile backbone structure determination of human membrane proteins by NMR spectroscopy. Nat. Methods 9 , 834-839.

Knowles, T.J., Finka, R., Smith, C., Lin, Y.P., Dafforn, T., and Overduin, M. (2009). Membrane proteins solubilized intact in lipid containing nanoparticles bounded by styrene maleic acid copolymer. J. Am. Chem. Soc. 131, 7484-7485.

Kobashigawa, Y., Harada, K., Yoshida, N., Ogura, K., and Inagaki, F. (2011). Phosphoinositide-incorporated lipid-protein nanodiscs: a tool for studying protein-lipid interactions. Anal. Biochem. 410, 77-83.

Kofuku, Y., Ueda, T., Okude, J., Shiraishi, Y., Kondo, K., Mizumura, T., Suzuki, S., and Shimada, I. (2014). Functional dynamics of 
deuterated $\beta 2$-adrenergic receptor in lipid bilayers revealed by NMR spectroscopy. Angew. Chem. Int. Ed. 53, 13376-13379.

Kucharska, I., Edrington, T.C., Liang, B., and Tamm, L.K. (2015). Optimizing nanodiscs and bicelles for solution NMR studies of two $\beta$-barrel membrane proteins. J. Biomol. NMR 61, 261-274.

Lee, T.Y., Yeh, V., Chuang, J., Chung Chan, J.C., Chu, L.K., and Yu, T.Y. (2015). Tuning the photocycle kinetics of bacteriorhodopsin in lipid nanodiscs. Biophys. J. 109, 1899-1906.

Lee, S.C., Knowles, T.J., Postis, V.L., Jamshad, M., Parslow, R.A., Lin, Y.P., Goldman, A., Sridhar, P., Overduin, M., Muench, S.P., et al. (2016). A method for detergent-free isolation of membrane proteins in their local lipid environment. Nat. Protoc. 11, 1149-1162.

Leitz, A., Bayburt, T., Barnakov, A., Springer, B., and Sligar, S. (2006). Functional reconstitution of $\beta 2$-adrenergic receptors utilizing self-assembling Nanodisc technology. BioTechniques 40, 601-612.

Leney, A.C., Fan, X., Kitova, E.N., and Klassen, J.S. (2014). Nanodiscs and electrospray ionization mass spectrometry: a tool for screening glycolipids against proteins. Anal. Chem. 86, 5271-5277.

Li, Y., Kijac, A.Z., Sligar, S.G., and Rienstra, C.M. (2006). Structural analysis of nanoscale self-assembled discoidal lipid bilayers by solid-state NMR spectroscopy. Biophys. J. 91, 3819-3828.

Liang, B. and Tamm, L.K. (2016). NMR as a tool to investigate the structure, dynamics and function of membrane proteins. Nat. Struct. Mol. Biol. 23, 468-474.

Long, A.R., O'Brien, C.C., Malhotra, K., Schwall, C.T., Albert, A.D., Watts, A., and Alder, N.N. (2013). A detergent-free strategy for the reconstitution of active enzyme complexes from native biological membranes into nanoscale discs. BMC Biotechnol. 13, 41.

Lyukmanova, E.N., Shenkarev, Z.O., Paramonov, A.S., Sobol, A.G., Ovchinnikova, T.V., Chupin, V.V., Kirpichnikov, M.P., Blommers, M.J., and Arseniev, A.S. (2008). Lipid-protein nanoscale bilayers: a versatile medium for NMR investigations of membrane proteins and membrane-active peptides. J. Am. Chem. Soc. 130, 2140-2141.

Lyukmanova, E.N., Shenkarev, Z.O., Khabibullina, N.F., Kopeina, G.S., Shulepko, M.A., Paramonov, A.S., Mineev, K.S., Tikhonov, R.V., Shingarova, L.N., Petrovskaya, L.E., et al. (2012). Lipid-protein nanodiscs for cell-free production of integral membrane proteins in a soluble and folded state: comparison with detergent micelles, bicelles and liposomes. Biochim. Biophys. Acta 1818, 349-358.

Mazhab-Jafari, M.T., Marshall, C.B., Stathopulos, P.B., Kobashigawa, Y., Stambolic, V., Kay, L.E., Inagaki, F., and Ikura, M. (2013). Membrane-dependent modulation of the mTOR activator Rheb: NMR observations of a GTPase tethered to a lipid-bilayer nanodisc. J. Am. Chem. Soc. 135, 3367-3370.

Mazhab-Jafari, M.T., Marshall, C.B., Smith, M.J., Gasmi-Seabrook, G.M., Stathopulos, P.B., Inagaki, F., Kay, L.E., Neel, B.G., and Ikura, M. (2015). Oncogenic and RASopathy-associated K-RAS mutations relieve membrane-dependent occlusion of the effector-binding site. Proc. Natl. Acad. Sci. USA 112, 6625-6630.

Mi, L.Z., Grey, M.J., Nishida, N., Walz, T., Lu, C., and Springer, T.A. (2008). Functional and structural stability of the epidermal growth factor receptor in detergent micelles and phospholipid nanodiscs. Biochemistry 47, 10314-10323.

Mineev, K.S., Goncharuk, S.A., Kuzmichev, P.K., Vilar, M., and Arseniev, A.S. (2015). NMR dynamics of transmembrane and intracellular domains of p75NTR in lipid-protein nanodiscs. Biophys. J. 109, 772-782.

Mitra, N., Liu, Y., Liu, J., Serebryany, E., Mooney, V., DeVree, B.T., Sunahara, R.K., Yan, E.C. (2013). Calcium-dependent ligand binding and G-protein signaling of family B GPCR parathyroid hormone 1 receptor purified in nanodiscs. ACS Chem. Biol. 8 , 617-625.

Morgado, L., Zeth, K., Burmann, B.M., Maier, T., and Hiller, S. (2015). Characterization of the insertase BamA in three different membrane mimetics by solution NMR spectroscopy. J. Biomol. NMR 61, 333-345.

Mors, K., Roos, C., Scholz, F., Wachtveitl, J., Dotsch, V., Bernhard, F., and Glaubitz, C. (2013). Modified lipid and protein dynamics in nanodiscs. Biochim. Biophys. Acta 1828, 1222-1229.

Nasvik Ojemyr, L., von Ballmoos, C., Gennis, R.B., Sligar, S.G., and Brzezinski, P. (2012). Reconstitution of respiratory oxidases in membrane nanodiscs for investigation of proton-coupled electron transfer. FEBS Lett. 586, 640-645.

Ni, D., Wang, Y., Yang, X., Zhou, H., Hou, X., Cao, B., Lu, Z., Zhao, X., Yang, K., and Huang, Y. (2014). Structural and functional analysis of the $\beta$-barrel domain of BamA from Escherichia coli. FASEB J. 28, 2677-2685.

Okude, J., Ueda, T., Kofuku, Y., Sato, M., Nobuyama, N., Kondo, K., Shiraishi, Y., Mizumura, T., Onishi, K., Natsume, M., et al. (2015). Identification of a conformational equilibrium that determines the efficacy and functional selectivity of the $\mu$-opioid receptor. Angew. Chem. Int. Ed. 54, 15771-15776.

Opella, S.J. (2013). Structure determination of membrane proteins by nuclear magnetic resonance spectroscopy. Annu. Rev. Anal. Chem. 6, 305-328.

Orwick, M.C., Judge, P.J., Procek, J., Lindholm, L., Graziadei, A., Engel, A., Grobner, G., and Watts, A. (2012). Detergent-free formation and physicochemical characterization of nanosized lipid-polymer complexes: Lipodisq. Angew. Chem. Int. Ed. 51, 4653-4657.

Orwick-Rydmark, M., Lovett, J.E., Graziadei, A., Lindholm, L., Hicks, M.R., and Watts, A. (2012). Detergent-free incorporation of a seven-transmembrane receptor protein into nanosized bilayer Lipodisq particles for functional and biophysical studies. Nano Lett. 12, 4687-4692.

Park, S.H., Berkamp, S., Cook, G.A., Chan, M.K., Viadiu, H., and Opella, S.J. (2011). Nanodiscs versus macrodiscs for NMR of membrane proteins. Biochemistry 50, 8983-8985.

Phillips, J.C., Wriggers, W., Li, Z., Jonas, A., and Schulten, K. (1997). Predicting the structure of apolipoprotein A-I in reconstituted high-density lipoprotein disks. Biophys. J. 73, 2337-2346.

Popot, J.L. (2010). Amphipols, nanodiscs, and fluorinated surfactants: three nonconventional approaches to studying membrane proteins in aqueous solutions. Annu. Rev. Biochem. 79, 737-775.

Postis, V., Rawson, S., Mitchell, J.K., Lee, S.C., Parslow, R.A., Dafforn, T.R., Baldwin, S.A., and Muench, S.P. (2015). The use of SMALPs as a novel membrane protein scaffold for structure study by negative stain electron microscopy. Biochim Biophys Acta 1848, 496-501.

Proverbio, D., Roos, C., Beyermann, M., Orban, E., Dotsch, V., and Bernhard, F. (2013). Functional properties of cell-free expressed human endothelin A and endothelin B receptors in artificial membrane environments. Biochim. Biophys. Acta 1828, 2182-2192.

Puthenveetil, R. and Vinogradova, O. (2013). Optimization of the design and preparation of nanoscale phospholipid bilayers for 
its application to solution NMR. Proteins: Struct. Funct. Bioinf. $81,1222-1231$.

Rajesh, S., Knowles, T., and Overduin, M. (2011). Production of membrane proteins without cells or detergents. Nat. Biotechnol. 28, 250-254.

Raschle, T., Hiller, S., Yu, T.Y., Rice, A.J., Walz, T., and Wagner, G. (2009). Structural and functional characterization of the integral membrane protein VDAC-1 in lipid bilayer nanodiscs. J. Am. Chem. Soc. 131, 17777-17779.

Raschle, T., Hiller, S., Etzkorn, M., and Wagner, G. (2010). Nonmicellar systems for solution NMR spectroscopy of membrane proteins. Curr. Opin. Struct. Biol. 20, 471-479.

Raschle, T., Lin, C., Jungmann, R., Shih, W.M., and Wagner, G. (2015). Controlled Co-reconstitution of multiple membrane proteins in lipid bilayer nanodiscs using DNA as a scaffold. ACS Chem. Biol. 10, 2448-2454.

Reichart, T.M., Baksh, M.M., Rhee, J.K., Fiedler, J.D., Sligar, S.G., Finn, M.G., Zwick, M.B., and Dawson, P.E. (2016). Trimerization of the HIV transmembrane domain in lipid bilayers modulates broadly neutralizing antibody binding. Angew. Chem. Int. Ed. $55,2688-2692$.

Ritchie, T.K., Grinkova, Y.V., Bayburt, T.H., Denisov, I.G., Zolnerciks, J.K., Atkins, W.M., and Sligar, S.G. (2009) Reconstitution of membrane proteins in phospholipid bilayer nanodiscs. In: Methods Enzymol., Vol. 464, Nejat. eds. (New York: Elsevier Inc.), pp. 211-231.

Roos, C., Zocher, M., Muller, D., Munch, D., Schneider, T., Sahl, H.G., Scholz, F., Wachtveitl, J., Ma, Y., Proverbio, D., et al. (2012). Characterization of co-translationally formed nanodisc complexes with small multidrug transporters, proteorhodopsin and with the E. coli MraY translocase. Biochim. Biophys. Acta 1818, 3098-3106.

Roos, C., Kai, L., Proverbio, D., Ghoshdastider, U., Filipek, S., Dotsch, V., and Bernhard, F. (2013). Co-translational association of cell-free expressed membrane proteins with supplied lipid bilayers. Mol. Membr. Biol. 30, 75-89.

Roy, J., Pondenis, H., Fan, T.M., and Das, A. (2015). Direct capture of functional proteins from mammalian plasma membranes into nanodiscs. Biochemistry 54, 6299-6302.

Rues, R.-B., Dötsch, V., and Bernhard, F. (2016). Co-translational formation and pharmacological characterization of beta1adrenergic receptor/nanodisc complexes with different lipid environments. Biochim. Biophys. Acta 1858, 1306-1316.

Salzmann, M., Pervushin, K., Wider, G., Senn, H., and Wuthrich, K. (1998). TROSY in triple-resonance experiments: new perspectives for sequential NMR assignment of large proteins. Proc. Natl. Acad. Sci. USA 95, 13585-13590.

Schuler, M.A., Denisov, I.G., and Sligar, S.G. (2013) Nanodiscs as a new tool to examine lipid-protein interactions. In: Lipid-Protein Interactions, Vol. 974, Kleinschmidt. eds. (New York: Springer), pp. 415-433.

Schwarz, D., Junge, F., Durst, F., Frolich, N., Schneider, B., Reckel, S., Sobhanifar, S., Dotsch, V., and Bernhard, F. (2007). Preparative scale expression of membrane proteins in Escherichia coli-based continuous exchange cell-free systems. Nat. Protoc. 2, 2945-2957.

Segrest, J.P. (1977). Amphipathic helixes and plasma lipoproteins: thermodynamic and geometric considerations. Chem. Phys. Lipids 18, 7-22.
Segrest, J.P., Jones, M.K., Klon, A.E., Sheldahl, C.J., Hellinger, M., De Loof, H., and Harvey, S.C. (1999). A detailed molecular belt model for apolipoprotein A-I in discoidal high density lipoprotein. J. Biol. Chem. 274, 31755-31758.

Shaw, A.W., McLean, M.A., and Sligar, S.G. (2004). Phospholipid phase transitions in homogeneous nanometer scale bilayer discs. FEBS Lett. 556, 260-264.

Shaw, A.W., Pureza, V.S., Sligar, S.G., and Morrissey, J.H. (2007). The local phospholipid environment modulates the activation of blood clotting. J. Biol. Chem. 282, 6556-6563.

Shenkarev, Z.O., Lyukmanova, E.N., Solozhenkin, O.I., Gagnidze, I.E., Nekrasova, O.V., Chupin, V.V., Tagaev, A.A., Yakimenko, Z.A., Ovchinnikova, T.V., Kirpichnikov, M.P., et al. (2009). Lipidprotein nanodiscs: possible application in high-resolution NMR investigations of membrane proteins and membrane-active peptides. Biochemistry (Moscow) 74, 756-765.

Shenkarev, Z.O., Lyukmanova, E.N., Paramonov, A.S., Shingarova, L.N., Chupin, V.V., Kirpichnikov, M.P., Blommers, M.J., and Arseniev, A.S. (2010a). Lipid-protein nanodiscs as reference medium in detergent screening for high-resolution NMR studies of integral membrane proteins. J. Am. Chem. Soc. 132, 5628-5629.

Shenkarev, Z.O., Paramonov, A.S., Lyukmanova, E.N., Shingarova, L.N., Yakimov, S.A., Dubinnyi, M.A., Chupin, V.V., Kirpichnikov, M.P., Blommers, M.J., and Arseniev, A.S. (2010b). NMR structural and dynamical investigation of the isolated voltage-sensing domain of the potassium channel KvAP: implications for voltage gating. J. Am. Chem. Soc. 132, 5630-5637.

Shenkarev, Z.O., Lyukmanova, E.N., Paramonov, A.S., Panteleev, P.V., Balandin, S.V., Shulepko, M.A., Mineev, K.S., Ovchinnikova, T.V., Kirpichnikov, M.P., and Arseniev, A.S. (2014). Lipidprotein nanodiscs offer new perspectives for structural and functional studies of water-soluble membrane-active peptides. Acta Naturae 6, 84-94.

Shi, L., Shen, Q.T., Kiel, A., Wang, J., Wang, H.W., Melia, T.J., Rothman, J.E., and Pincet, F. (2012). SNARE proteins: one to fuse and three to keep the nascent fusion pore open. Science 335, 1355-1359.

Shi, L., Howan, K., Shen, Q.T., Wang, Y.J., Rothman, J.E., and Pincet, F. (2013). Preparation and characterization of SNARE-containing nanodiscs and direct study of cargo release through fusion pores. Nat. Protoc. 8, 935-948.

Shih, A.Y., Denisov, I.G., Phillips, J.C., Sligar, S.G., and Schulten, K. (2005). Molecular dynamics simulations of discoidal bilayers assembled from truncated human lipoproteins. Biophys. J. 88, 548-556.

Shih, A.Y., Arkhipov, A., Freddolino, P.L., Sligar, S.G., and Schulten, K. (2007a). Assembly of lipids and proteins into lipoprotein particles. J. Phys. Chem. B 111, 11095-11104.

Shih, A.Y., Freddolino, P.L., Arkhipov, A., and Schulten, K. (2007b). Assembly of lipoprotein particles revealed by coarse-grained molecular dynamics simulations. J. Struct. Biol. 157, 579-592.

Shih, A.Y., Sligar, S.G., and Schulten, K. (2009). Maturation of highdensity lipoproteins. J. R. Soc. Interface 6, 863-871.

Skar-Gislinge, N., Simonsen, J.B., Mortensen, K., Feidenhans'l R., Sligar, S.G., Lindberg Moller, B., Bjornholm, T., and Arleth, L. (2010). Elliptical structure of phospholipid bilayer nanodiscs encapsulated by scaffold proteins: casting the roles of the lipids and the protein. J. Am. Chem. Soc. 132, 13713-13722. 
Susac, L., Horst, R., and Wuthrich, K. (2014). Solution-NMR characterization of outer-membrane protein A from E. coli in lipid bilayer nanodiscs and detergent micelles. ChemBioChem 15, 995-1000.

Thomas, M.J., Bhat, S., and Sorci-Thomas, M.G. (2008). Three-dimensional models of HDL apoA-I: implications for its assembly and function. J. Lipid Res. 49, 1875-1883.

Tsukamoto, H., Sinha, A., DeWitt, M., and Farrens, D.L. (2010). Monomeric rhodopsin is the minimal functional unit required for arrestin binding. J. Mol. Biol. 399, 501-511.

Tzitzilonis, C., Eichmann, C., Maslennikov, I., Choe, S., and Riek, R. (2013). Detergent/nanodisc screening for high-resolution NMR studies of an integral membrane protein containing a cytoplasmic domain. PLoS One 8, e54378.

Viegas, A., Viennet, T., Yu, T.Y., Schumann, F., Bermel, W., Wagner, G., and Etzkorn, M. (2016). UTOPIA NMR: activating unexploited magnetization using interleaved low-gamma detection. J. Biomol. NMR 64, 9-15.

Vilar, M., Charalampopoulos, I., Kenchappa, R.S., Simi, A., Karaca, E., Reversi, A., Choi, S., Bothwell, M., Mingarro, I., Friedman, W.J., Schiavo, G., Bastiaens, P.I., Verveer, P.J., Carter, B.D., and Ibanez, C.F. (2009). Activation of the p75 neurotrophin receptor through conformational rearrangement of disulphide-linked receptor dimers. Neuron 62, 72-83.

Vogt, J. and Schulz, G.E. (1999). The structure of the outer membrane protein OmpX from Escherichia coli reveals possible mechanisms of virulence. Structure 7, 1301-1309.

Wan, C., Wu, B., Song, Z., Zhang, J., Chu, H., Wang, A., Liu, Q., Shi, Y., Li, G., and Wang, J. (2015). Insights into the molecular recognition of the granuphilin $\mathrm{C} 2 \mathrm{~A}$ domain with $\mathrm{PI}(4,5) \mathrm{P}_{2}$. Chem. Phys. Lipids 186, 61-67.

Wang, X., Mu, Z., Li, Y., Bi, Y., and Wang, Y. (2015). Smaller Nanodiscs are Suitable for Studying Protein Lipid Interactions by Solution NMR. Protein, J. 34, 205-211.

Whorton, M.R., Bokoch, M.P., Rasmussen, S.G., Huang, B., Zare, R.N., Kobilka, B., and Sunahara, R.K. (2007). A monomeric $G$ protein-coupled receptor isolated in a high-density lipoprotein particle efficiently activates its $\mathrm{G}$ protein. Proc. Natl. Acad. Sci. USA 104, 7682-7687.

Wu, Z., Wagner, M.A., Zheng, L., Parks, J.S., Shy, J.M., 3rd, Smith, J.D., Gogonea, V., and Hazen, S.L. (2007). The refined structure of nascent HDL reveals a key functional domain for particle maturation and dysfunction. Nat. Struct. Mol. Biol. 14, 861-868.

Wu, Z., Gogonea, V., Lee, X., Wagner, M.A., Li, X.M., Huang, Y., Undurti, A., May, R.P., Haertlein, M., Moulin, M., Gutsche, I., Zaccai, G., Didonato, J.A., and Hazen, S.L. (2009). Double superhelix model of high density lipoprotein. J. Biol. Chem. 284, 36605-36619.

Yang, J.P., Cirico, T., Katzen, F., Peterson, T.C., and Kudlicki, W. (2011). Cell-free synthesis of a functional G protein-coupled receptor complexed with nanometer scale bilayer discs. BMC Biotechnol. 11, 57.

Yao, Y., Fujimoto, L.M., Hirshman, N., Bobkov, A.A., Antignani, A., Youle, R.J., and Marassi, F.M. (2015). Conformation of BCL-XL upon membrane integration. J. Mol. Biol. 427, 2262-2270.

Yao, Y., Nisan, D., Fujimoto, L.M., Antignani, A., Barnes, A., Tjandra, N., Youle, R.J., and Marassi, F.M. (2016). Characterization of the membrane-inserted C-terminus of cytoprotective BCL-XL. Protein Expr. Purif. 122, 56-63.

Yokogawa, M., Kobashigawa, Y., Yoshida, N., Ogura, K., Harada, K., and Inagaki, F. (2012). NMR analyses of the interaction between the FYVE domain of early endosome antigen 1 (EEA1) and phosphoinositide embedded in a lipid bilayer. J. Biol. Chem. 287, 34936-34945.

Yoshiura, C., Kofuku, Y., Ueda, T., Mase, Y., Yokogawa, M., Osawa, M., Terashima, Y., Matsushima, K., and Shimada, I. (2010). NMR analyses of the interaction between CCR5 and its ligand using functional reconstitution of CCR5 in lipid bilayers. J. Am. Chem. Soc. 132, 6768-6777.

Yu, T.Y., Raschle, T., Hiller, S., and Wagner, G. (2012). Solution NMR spectroscopic characterization of human VDAC-2 in detergent micelles and lipid bilayer nanodiscs. Biochim. Biophys. Acta 1818, 1562-1569.

Zhang, Z., Dai, C., Bai, J., Xu, G., Liu, M., and Li, C. (2014). Ca ${ }^{2+}$ modulating $\alpha$-synuclein membrane transient interactions revealed by solution NMR spectroscopy. Biochim. Biophys. Acta 1838, 853-858.

Zhang, M., Huang, R., Ackermann, R., Im, S.C., Waskell, L., Schwendeman, A., and Ramamoorthy, A. (2016). Reconstitution of the Cytb5 -CytP450 complex in nanodiscs for structural studies using NMR spectroscopy. Angew. Chem. Int. Ed. 55, 4497-4499. 